\title{
Radio emission models of colliding-wind binary systems
}

\author{
S. M. Dougherty ${ }^{1}$, J. M. Pittard ${ }^{2}$, L. Kasian ${ }^{1}$, R. F. Coker $^{3}$, P. M. Williams ${ }^{4}$, and H. M. Lloyd ${ }^{5}$ \\ ${ }^{1}$ National Research Council of Canada, Herzberg Institute for Astrophysics, Dominion Radio Astrophysical Observatory, \\ PO Box 248, Penticton, British Columbia V2A 6J9, Canada \\ 2 Department of Physics and Astronomy, The University of Leeds, Woodhouse Lane, Leeds LS2 9JT, UK \\ 3 Los Alamos National Laboratory, X-2 MS T-087, Los Alamos, NM 87545, USA \\ ${ }^{4}$ Institute for Astronomy, University of Edinburgh, Royal Observatory, Blackford Hill, Edinburgh EH9 3HJ, UK \\ 5 Blade Interactive Studios, 274 Deansgate, Manchester M3 4JB, UK
}

Received 25 April 2003 / Accepted 7 July 2003

\begin{abstract}
We present calculations of the spatial and spectral distribution of the radio emission from a wide WR $+\mathrm{OB}$ collidingwind binary system based on high-resolution hydrodynamical simulations and solutions to the radiative transfer equation. We account for both thermal and synchrotron radio emission, free-free absorption in both the unshocked stellar wind envelopes and the shocked gas, synchrotron self-absorption, and the Razin effect. To calculate the synchrotron emission several simplifying assumptions are adopted: the relativistic particle energy density is a simple fraction of the thermal particle energy density, in equipartition with the magnetic energy density, and a power-law in energy. We also assume that the magnetic field is tangled such that the resulting emission is isotropic. The applicability of these calculations to modelling radio images and spectra of colliding-wind systems is demonstrated with models of the radio emission from the wide WR+OB binary WR 147 . Its synchrotron spectrum follows a power-law between 5 and $15 \mathrm{GHz}$ but turns down to below this at lower and higher frequencies. We find that while free-free opacity from the circum-binary stellar winds can potentially account for the low-frequency turnover, models that also include a combination of synchrotron self-absorption and Razin effect are favoured. We argue that the highfrequency turn down is a consequence of inverse-Compton cooling. We present our resulting spectra and intensity distributions, along with simulated MERLIN observations of these intensity distributions. From these we argue that the inclination of the WR 147 system to the plane of the sky is low. We summarise by considering extensions of the current model that are important for models of the emission from closer colliding wind binaries, in particular the dramatically varying radio emission of WR 140 .
\end{abstract}

Key words. stars: binaries: general - stars: early-type - stars: individual: WR 147 - stars: Wolf-Rayet - radio continuum: stars

\section{Introduction}

Observations of early-type stars have revealed that they can be sources of both thermal and synchrotron radio emission. Thermal emission typically exhibits a brightness temperature $\sim 10^{4} \mathrm{~K}$ and a spectral index $\alpha \sim+0.6\left(S_{v} \propto v^{\alpha}\right)$ at centimetre wavelengths, as expected from steady-state, radially symmetric winds (Wright \& Barlow 1975). In contrast, synchrotron emission is characterized by high brightness temperatures $\left(\geq 10^{6} \mathrm{~K}\right)$ and flat or negative radio spectral indices. In addition to magnetic fields, synchrotron emission requires a population of relativistic electrons, which are widely thought to be accelerated in shocks. For single stars, shocks arise due to wind instabilities e.g. Lucy \& White (1980), and propagate through the wind, while for massive binary systems stationary shocks arise where the winds of the two stars collide e.g. Eichler \& Usov (1993). Magnetic field compression within the shocked gas is another potential acceleration mechanism (Jardine et al. 1996).

Send offprint requests to: S. M. Dougherty, e-mail: sean.dougherty@nrc.gc.ca
Spatially resolved observations of the $\mathrm{WR}+\mathrm{OB}$ binary systems WR 146 (Dougherty et al. 1996, 2000) and WR 147 (Moran et al. 1989; Churchwell et al. 1992; Williams et al. 1997; Niemela et al. 1998) have dramatically confirmed the colliding-wind binary (CWB) model in these objects. In both WR 146 and WR 147 the thermal emission is coincident with the position of the WR star, while the synchrotron emission arises between the binary components at a position consistent with the pressure balance of the two stellar winds. This model is supported further by the dramatic variations of the synchrotron radio emission in the 7.9-year WR+OB system WR 140, which are clearly modulated by the binary orbit e.g. Williams et al. (1990a); White \& Becker (1995).

Such results prompted van der Hucht et al. (1992) to suggest a CWB origin for all synchrotron emission from WR stars, for which there is now strong observational support (Dougherty \& Williams 2000). It is possible that this is also the case in O-type stars with synchrotron radio emission, e.g. Cyg OB2 \#5 (Contreras et al. 1997), though there remain several apparently single stars that obviously do not fit a CWB interpretation.

To date, modelling has been restricted to the radiometry from such systems. At frequency $v$, the observed flux $\left(S_{v}^{\text {obs }}\right)$ is 
related to the thermal flux $\left(S_{v}^{\text {th }}\right)$, the synchrotron emission arising from the wind-wind collision region $\left(S_{v}^{\mathrm{syn}}\right)$, and the freefree opacity $\left(\tau_{v}^{\mathrm{ff}}\right)$ of the circum-system stellar wind envelope by

$S_{v}^{\text {obs }}=S_{v}^{\text {th }}+S_{v}^{\text {syn }} \mathrm{e}^{-\tau_{v}^{\mathrm{ff}}}$.

It is typically assumed that the stellar wind envelope is radially symmetric and that the synchrotron emission arises from a point source at the stagnation point of the two stellar winds (see Williams et al. 1990a; Chapman et al. 1999; Skinner et al. 1999; Monnier et al. 2002 for examples). In this manner, relatively simple analytical solutions to the radiative transfer equation can be obtained. Although these models may successfully recover the radiometry, a single-valued free-free opacity determined along the line-of-sight to a point-like synchrotron emission region is an over-simplification, especially considering the extended region of synchrotron emission observed from both WR 146 and WR 147. Furthermore, in a CWB the assumption of radial symmetry fails in the collision zone.

So far, no attempts have been made to construct synthetic radio images based on more realistic density and temperature distributions. This is surely imperative given the advent of spatially resolved observations where direct comparison between models and observations may be expected to increase our understanding of this phenomenon. In making the first steps toward addressing this situation, we have calculated the free-free and synchrotron radio emission arising from an early-type binary system under various simplifying assumptions. Our approach extends and improves on previous work by including the ability to simulate both the free-free and synchrotron emission and absorption from the stellar winds and wind-wind collision region based on 2D, axis-symmetric hydrodynamical simulations of the density and pressure distribution. Various synchrotron cooling mechanisms can also be incorporated as required. An arbitrary inclination angle for the line of sight can be specified, and the radiative transfer equation is then solved to generate synthetic images and spectra.

The layout of the remainder of this paper is as follows. In Sect. 2 we describe our model in more detail and in Sect. 3 we perform a parameter study using a "standard" model of a very wide CWB. We examine the influence of synchrotron self-absorption and the Razin effect, which have not hitherto received much attention, along with system inclination and binary separation. The application of our model to observations of WR 147 is described in Sect. 4. In Sect. 5 we summarize and note future directions. In Appendix A, the geometry of the raytracing technique for solving the radiative transfer equation is described.

\section{Modelling the radio emission from CWBs}

To calculate the radio emission from an early-type binary, we must first compute the structure of the wind-wind collision. This is most readily achieved through the use of a hydrodynamical code, and we use VH-1 (Blondin et al. 1990), a Lagrangian remap version of the piecewise-parabolic method e.g. Pittard $\&$ Stevens (1997). In this paper we are concerned particularly with wide systems, so it is assumed that the spherically symmetric stellar winds are accelerated instantaneously to terminal speeds. This is reasonable given that the winds attain terminal velocity at a small percentage of the radial distance from the stars to the wind collision region. Distortion of the collision zone by orbital motion, most especially close to the shock apex, is negligible so axis-symmetry is also assumed. We use WR or solar abundances for the stellar winds, as appropriate. Such models have been successfully applied to the analyses of X-ray observations from CWBs (see Pittard \& Corcoran 2002; Pittard et al. 2002 and references therein).

Once a suitable hydrodynamic solution has been obtained, the necessary information is read into our radiative transfer raytracing code, and appropriate emission and absorption coefficients for each cell on the 2D grid are determined. A synthetic image on the plane of the sky is then generated by solving the radiative transfer equation along suitable lines of sight through the grid (see Appendix A for more details). The following sections detail the calculation of the emission and absorption properties for each hydrodynamic cell.

\subsection{Thermal emission and absorption}

The thermal emission $\left(\varepsilon_{v}^{\mathrm{ff}}\right)$ and absorption $\left(\alpha_{v}^{\mathrm{ff}}\right)$ coefficients at a frequency $v$ are given by Rybicki \& Lightman (1979) as

$\varepsilon_{v}^{\mathrm{ff}}=6.8 \times 10^{-38} Z^{2} n_{\mathrm{e}} n_{\mathrm{i}} T^{-1 / 2} \mathrm{e}^{-h v / k T} g_{\mathrm{ff}}$,

$\alpha_{v}^{\mathrm{ff}}=3.7 \times 10^{8} T^{-1 / 2} Z^{2} n_{\mathrm{e}} n_{\mathrm{i}} v^{-3}\left(1-\mathrm{e}^{-h v / k T}\right) g_{\mathrm{ff}}$,

where $Z$ is the ionic charge, $n_{\mathrm{e}}$ and $n_{\mathrm{i}}$ are electron and ion number densities, $T$ is the temperature of the gas, and $g_{\mathrm{ff}}$ is a velocity averaged Gaunt factor (Hummer 1988). The densities $n_{\mathrm{e}}$ and $n_{\mathrm{i}}$ are determined from the cell density, composition and ionization, where the ionization is specified as a function of cell temperature. The appropriate ionization of the elemental species for a particular temperature regime is determined and the absorption and emission coefficients are then evaluated for several ionic charges.

The accuracy of the "thermal" code was verified by using a simple spherically symmetric, isothermal stellar wind model with an $r^{-2}$ radial density distribution. The resulting fluxes and free-free opacities matched those derived from the analytical expressions given in Wright \& Barlow (1975).

\subsection{Synchrotron emission}

When non-relativistic particles are accelerated in a magnetic field, emission occurs at frequencies corresponding to harmonics of the gyration frequency of the particle. This is known as cyclotron emission, and most of the radiated power occurs at the gyration frequency. On the other hand, if the particles are relativistic, the emission blurs into a continuum rather than a series of delta functions and emission may occur at a frequency many orders of magnitude greater than the gyration frequency. The synchrotron emission from a single electron is (cf. Rybicki \& Lightman 1979)

$P(v)=\frac{\sqrt{3} q^{3} B \sin \alpha}{m c^{2}} F\left(v / v_{\mathrm{c}}\right)$

where $q$ is the particle charge, $B$ is the magnetic field strength, $\alpha$ is the pitch angle of the particle relative to the direction of the 
$B$-field, $m$ is the mass of the particle, and $F\left(v / v_{\mathrm{c}}\right)$ is a dimensionless function describing the total power spectrum of the synchrotron emission, with $v_{\mathrm{c}}$ the frequency where the spectrum cuts off, given by

$v_{c}=\frac{3 \gamma^{2} q B \sin \alpha}{4 \pi m c}$.

Values for $F\left(v / v_{\mathrm{c}}\right)$ are tabulated in Ginzburg \& Syrovatskii (1965). Throughout the remainder of this paper we assume that $\sin \alpha=1$. The emissivity per unit volume from a monoenergetic distribution of electrons is then simply the product of Eq. (4) and the number density of the radiating particles.

In our model we suppose that the relativistic electrons are created by 1 st-order Fermi acceleration at the shocks where the winds collide. For a strong shock with a compression ratio of 4 , test particle theory predicts that accelerated electrons will have an energy distribution with a power-law index of 2 (see references in Eichler \& Usov 1993). Hence, the number of relativistic electrons per unit volume with energies between $\gamma$ and $\gamma+\mathrm{d} \gamma$ is $N(\gamma) \mathrm{d} \gamma=C \gamma^{-p} \mathrm{~d} \gamma$, where $\gamma$ is the Lorentz factor and $p=2$. More recent nonlinear calculations yield a similar energy spectrum for a wide range of shock parameters e.g. Ellison \& Eichler (1985). A useful discussion of shock acceleration can be found in Ellison \& Reynolds (1991).

For such a power-law distribution of electrons, the total synchrotron emission at frequency $v$ is given by (Rybicki \& Lightman 1979)

$$
\begin{aligned}
P(v)= & \frac{\sqrt{3} q^{3} C B}{m c^{2}(p+1)} \Gamma\left(\frac{p}{4}+\frac{19}{12}\right) \Gamma\left(\frac{p}{4}-\frac{1}{12}\right) \\
& \times\left(\frac{2 \pi m c v}{3 q B}\right)^{-(p-1) / 2},
\end{aligned}
$$

where $\Gamma$ is the Gamma function. Equation (6) is only strictly valid in the synchrotron limit, when the frequency of emission is between approximately a few times the gyration frequency $\left(v_{B}\right)$ and $v_{\mathrm{c}}$ (defined in Eq. (10)), but we restrict this investigation to frequencies much greater than $v_{B}$ e.g. for our model of WR $147 v_{B}<10^{4} \mathrm{~Hz}$ at the shock apex.

To evaluate Eq. (6), we must determine the appropriate value for the normalization constant $C$, and the magnetic field strength $B$. Since our hydrodynamical simulations do not provide direct information on the magnetic field or relativistic particle distribution, we follow the standard procedure in such cases (e.g. Chevalier 1982; Mioduszewski et al. 2001): that is, to assume that the magnetic energy density $U_{B}$, and the relativistic particle energy density $U_{\text {rel }}$, are proportional to the thermal particle internal energy density $U_{\text {th }}$. Then

$U_{B}=B^{2} / 8 \pi=\zeta_{B} U_{\mathrm{th}}$,

$U_{\text {rel }}=\int n(\gamma) \gamma m c^{2} \mathrm{~d} \gamma=\zeta_{\text {rel }} U_{\text {th }}$,

where $\zeta_{B}$ and $\zeta_{\text {rel }}$ are constants which are typically set to around $1 \%$ (Mioduszewski et al. 2001), and $U_{\text {th }}=\frac{3}{2} P$ where $P$ is the gas pressure and the adiabatic index of $5 / 3$ is assumed, as for an ideal gas. The common assumption of equipartition corresponds to $\zeta_{B}=\zeta_{\text {rel }}=\zeta$.
As noted in Mioduszewski et al. (2001), there is some justification for such a relation between the energy densities: the thermal energy density is higher in the post-shock than in the pre-shock gas, and this is also where the magnetic field is likely to be enhanced, and the particles accelerated to relativistic energies. However, such arguments ultimately need to be replaced by detailed physical processes. It is well established that the shock acceleration of protons is very efficient, but how much energy is transferred to electrons in shocks is much less certain. We note that the relativistic electron energy is unlikely to be more than $5 \%$ of the total available shock energy, and could conceivably be much less (Blandford \& Eichler 1987; Ellison \& Reynolds 1991; Eichler \& Usov 1993).

$$
\text { For } p=2 \text {, }
$$

$C=\frac{U_{\text {rel }}}{m_{\mathrm{e}} c^{2} \ln \gamma_{\max }}$

where we have assumed $1<\gamma<\gamma_{\max }$ and $\gamma_{\max }$ specifies the maximum energy of the relativistic electrons. Since it is assumed in Eq. (6) that $\gamma_{\max } \rightarrow \infty$, the synchrotron emission extends to infinitely high frequencies with a spectrum $\propto v^{-0.5}$. In reality the synchrotron flux will depart from this relationship at frequencies near $v_{\mathrm{c}}$, where $v_{\mathrm{c}}$ is the frequency of peak emission from a particle of energy $E=\gamma_{\max } m c^{2}$, given by (Rybicki \& Lightman 1979)

$v_{\mathrm{c}} \approx \frac{3}{4 \pi} \frac{\gamma_{\max }^{2} q B}{m c}$

In CWBs we expect $\gamma_{\max }$ to be set by the balance between energy gain by Fermi acceleration and various cooling processes, which include inverse-Compton (hereafter IC) cooling, synchrotron decay, and ion-neutral wave damping. Ion-neutral damping does not play a dominant role in CWBs (Eichler \& Usov 1993), and is not discussed further here. The relevant processes and timescales are discussed in the following subsections.

\subsection{Synchrotron self-absorption}

We include synchrotron self-absorption (hereafter SSA) in our models. For a power-law distribution of particle energies, the absorption coefficient for SSA is (Rybicki \& Lightman 1979)

$$
\begin{aligned}
& \alpha_{v}=\frac{\sqrt{3} q^{3}}{8 \pi m}\left(\frac{3 q}{2 \pi m^{3} c^{5}}\right)^{p / 2} C B^{(p+2) / 2} \\
& \Gamma\left(\frac{3 p+12}{12}\right) \Gamma\left(\frac{3 p+22}{12}\right) v^{-(p+4) / 2}
\end{aligned}
$$

where the symbols have their previously defined meanings.

\subsection{The Razin effect}

When relativistic charges are surrounded by a plasma (as opposed to existing in a vacuum), the beaming effect that characterizes synchrotron radiation is suppressed. In essence, 
the refractive index of the medium reduces the Lorentz factor of the charge to

$$
\gamma^{\prime}=\frac{\gamma}{\sqrt{1+\gamma^{2} v_{0}^{2} / v^{2}}}
$$

where the plasma frequency $v_{0}=\sqrt{q^{2} n / \pi m}$. Hence synchrotron emission by relativistic charges in the medium is possible only when $\gamma^{\prime} \gg 1$, and a low frequency cut-off occurs. Quantitative details of this process, which is known as the Tsytovitch-Eidman-Razin effect, can be found in Hornby \& Williams (1966). The characteristic cut-off frequency is given by

$v_{\mathrm{R}}=20 \frac{n_{\mathrm{e}}}{B}$.

Since the emission decreases exponentially at frequencies low compared with $v_{\mathrm{R}}$, and there is a noticeable effect at frequencies greater than $v_{\mathrm{R}}$, e.g. there is a $10 \%$ reduction in flux at $v=10 v_{\mathrm{R}}$, we approximate this reduction by multiplying the synchrotron flux from our model by a factor $\mathrm{e}^{-\nu_{\mathrm{R}} / v}$.

\subsection{The low and high-energy cutoff in the relativistic particle distribution}

The nature of the synchrotron spectrum is determined by the underlying relativistic particle energy distribution. The low and high energy cutoffs in the energy distribution are particularly important. At low energies, the relativistic particle distribution can be potentially affected by coulombic collisions with thermal ions. However, at the densities and temperatures in the wind-collision regions of the wide systems considered in this paper this process is insignificant. The high energy cutoff is determined at the point where the rate of energy gain balances the rate of energy loss. Radiation losses due to synchrotron emission and IC scattering are in the same ratio as the magnetic field energy density to the photon energy density,

$$
\frac{P_{\mathrm{sync}}}{P_{\mathrm{compt}}}=\frac{U_{B}}{U_{\mathrm{ph}}} .
$$

As noted in Sect. 2.2, it is expected that $\zeta_{B}$ is no more than 0.05 , which suggests that $P_{\text {sync }}<P_{\text {compt }}$ in the wide CWB systems under consideration, and IC cooling dominates over synchrotron losses.

Electrons are rapidly accelerated to relativistic energies at the shocks bounding the wind-collision zone, and attain an energy spectrum specified by $p=2$ and $\gamma_{\max }$ (where the rate of energy loss by IC cooling balances the rate of energy gain from 1st order Fermi acceleration). They are then advected out of the shock and (for $\gamma<10^{5}$ ) are effectively frozen into the post-shock flow (White 1985). If the characteristic flow time out of the system is shorter than the timescale for IC cooling, the dominant cooling process is adiabatic expansion. This is the case for wide CWBs and low $\gamma$, and since adiabatic cooling is treated by the hydrodynamic code, we assume that $\zeta_{B}$ and $\zeta_{\text {rel }}$ are spatially invariant in the post-shock flow.
For high $\gamma$, IC losses can be very rapid even in the widest systems, as we show is the case for WR 147 in Sect. 4. IC cooling will be an even more important consideration in closer CWBs e.g. WR 140 and shorter period systems, where it could prevent the acceleration of electrons to even fairly modest values of $\gamma$. In these first attempts at modelling the radio emission from wide CWBs we have not included IC cooling explicitly in the code, but where relevant we discuss its impact on the resulting spectra e.g. Sects. 3.3 and 4. This will be treated more fully in Pittard et al. (in preparation).

\subsection{Some simplifying assumptions}

For simplicity in this first investigation, we assume that the post-shock ion and electron temperatures equalize very rapidly. While the equilibration timescale can be significant compared with the flow timescale e.g. WR 140 - see Usov (1992); Zhekov \& Skinner (2000), the exact situation is currently unclear with the importance of possible electron heating mechanisms in collision-less shocks still being debated. We anticipate that our simplification will have little bearing on the resulting radio emission because the synchrotron emission from the windcollision zone dominates the thermal free-free emission. While it may affect the efficiency with which the accelerated electrons are extracted from the thermal pool, this conjecture is impossible to test currently. Instead its effect is simply folded into the value of $\zeta$. Also, we assume that the gas is in ionization equilibrium, which is not most likely the case immediately postshock, especially in wide systems. However, we believe that this will not impact the results presented here since the bulk of the emission from the shocked gas is synchrotron, not free-free emission.

One final assumption in our models is that the magnetic field in the post-shock gas is highly tangled. Conveniently, this allows us to treat the synchrotron emission and absorption as isotropic, and means that it is possible to use isotropic formulae to calculate the IC losses (Pittard et al., in preparation). While the magnetic field from each star should be structured on large scales, and toroidal in shape at the large distances considered in the models presented here (see Eichler \& Usov 1993, and references therein), one might expect finer-scale structure in the post-shock flow. However, such details are beyond the scope of this paper.

\section{Parameter study}

\subsection{A standard model}

Our initial investigations have been based around a standard CWB model with the following parameters: $\dot{M}_{\mathrm{WR}}=2 \times$ $10^{-5} M_{\odot} \mathrm{yr}^{-1}, \dot{M}_{\mathrm{OB}}=2 \times 10^{-6} M_{\odot} \mathrm{yr}^{-1}, v_{\infty, \mathrm{WR}}=v_{\infty, \mathrm{OB}}=$ $2000 \mathrm{~km} \mathrm{~s}^{-1}, D=2 \times 10^{15} \mathrm{~cm}$, and $T_{\text {wind }}$ for both stars of $20 \mathrm{kK}$. These wind values are typical of WR and OB stars. At a frequency $v=5 \mathrm{GHz}$, the adopted binary separation $D$, is approximately $10 \times$ the radius of the $\tau_{\mathrm{ff}}=1$ surface of the WR wind. For inclination angles $\sim 0^{\circ}$, the lines of sight to the wind-collision zone are then optically thin, permitting investigation of the resulting emission from the wind-collision 


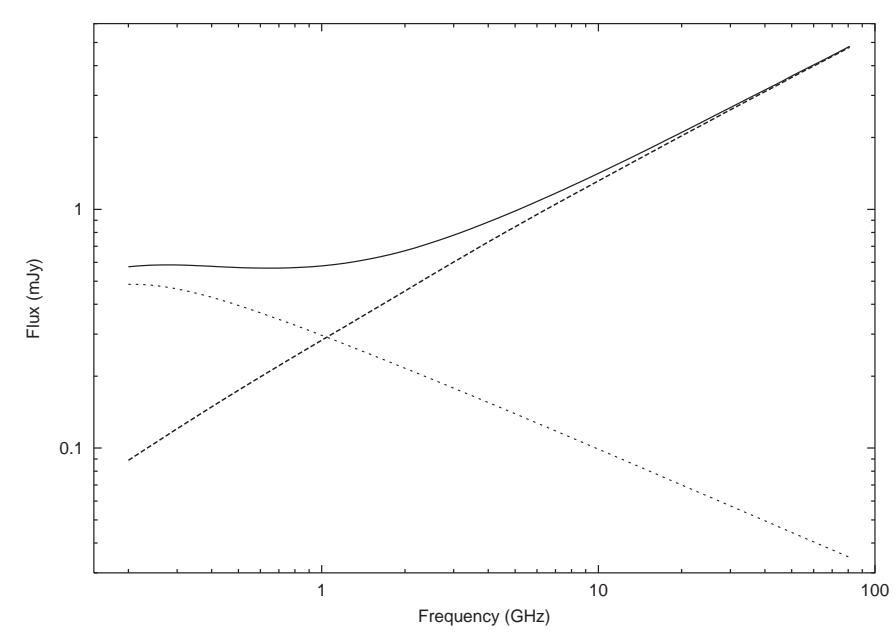

Fig. 1. Spectra from our standard model with $0^{\circ}$ inclination - free-free flux (dashed), synchrotron flux (dotted), and the total flux (solid). The effect of free-free absorption on the synchrotron emission in this wide system is only just evident below $300 \mathrm{MHz}$. The spectral index of the thermal spectrum is +0.6 , and the optically thin part of the synchrotron spectrum is -0.5 . Neither the Razin effect nor SSA are treated in this model.

region in the absence of strong free-free absorption from the circum-binary stellar wind envelope. We also assume solar abundances for the $\mathrm{OB}$ star and mass fractions $X=0, Y=$ $0.75, Z=0.25$ appropriate for a WC-type star, and an ionization structure of $\mathrm{H}^{+}, \mathrm{He}^{+}$and $\mathrm{CNO}^{2+}$. We adopt $\zeta=10^{-4}$ as our "canonical" value of $U_{B} / U_{\text {th }}$ and place our model system at a distance of $1.0 \mathrm{kpc}$. Maximum values of temperature, density, and magnetic field occur at the shock apex and for these input parameters we find $T_{\max }=1.75 \times 10^{8} \mathrm{~K}$, $\rho_{\text {max }}=9 \times 10^{-19} \mathrm{~g} \mathrm{~cm}^{-3}\left(n_{\max }=4 \times 10^{5} \mathrm{~cm}^{-3}\right)$, and $B_{\max }=6 \mathrm{mG}$. Though $B$ is an equipartition value, fields of this order at the location of the shock extrapolate to surface fields on the OB star of $\sim 30-300 \mathrm{G}$, using the magnetic field structure given in Eichler \& Usov (1993) and taking the typical rotation speed of O-type stars to be $\sim 0.1 v_{\infty, \mathrm{OB}}$ (Conti \& Ebbets 1977). Such surface fields are consistent with the few observations of the fields in these types of stars (Donati et al. $2001,2002)$. For all of the models presented in this paper we fix $\gamma_{\max }=4000$. Since we are using Eq. (6) to calculate the synchrotron luminosity, fixing $\gamma_{\max }$ only has a weak impact on the constant $C \sim 1 / \ln \gamma_{\max }$.

The radiometry and intensity distributions obtained from our standard model are shown in Figs. 1 and 2. In these first examples we only consider the impact of free-free absorption: SSA and the Razin effect are examined in the next section. Around $1 \mathrm{GHz}$ the synchrotron emission from the windwind collision is comparable to the total free-free emission, while at $22 \mathrm{GHz}$ the emission is largely from the two stellar winds. Below $1 \mathrm{GHz}$, the synchrotron emission dominates the total flux and the beginnings of a turnover seen at $\sim 300 \mathrm{MHz}$ in the synchrotron spectrum is due to free-free absorption from the unshocked stellar winds. Above $300 \mathrm{MHz}$, the synchrotron spectrum is a power-law with a spectral index equal to $(1-p) / 2=-0.5$ for $p=2$. The thermal spectrum is also a power-law with a spectral index of +0.6 , as expected for a

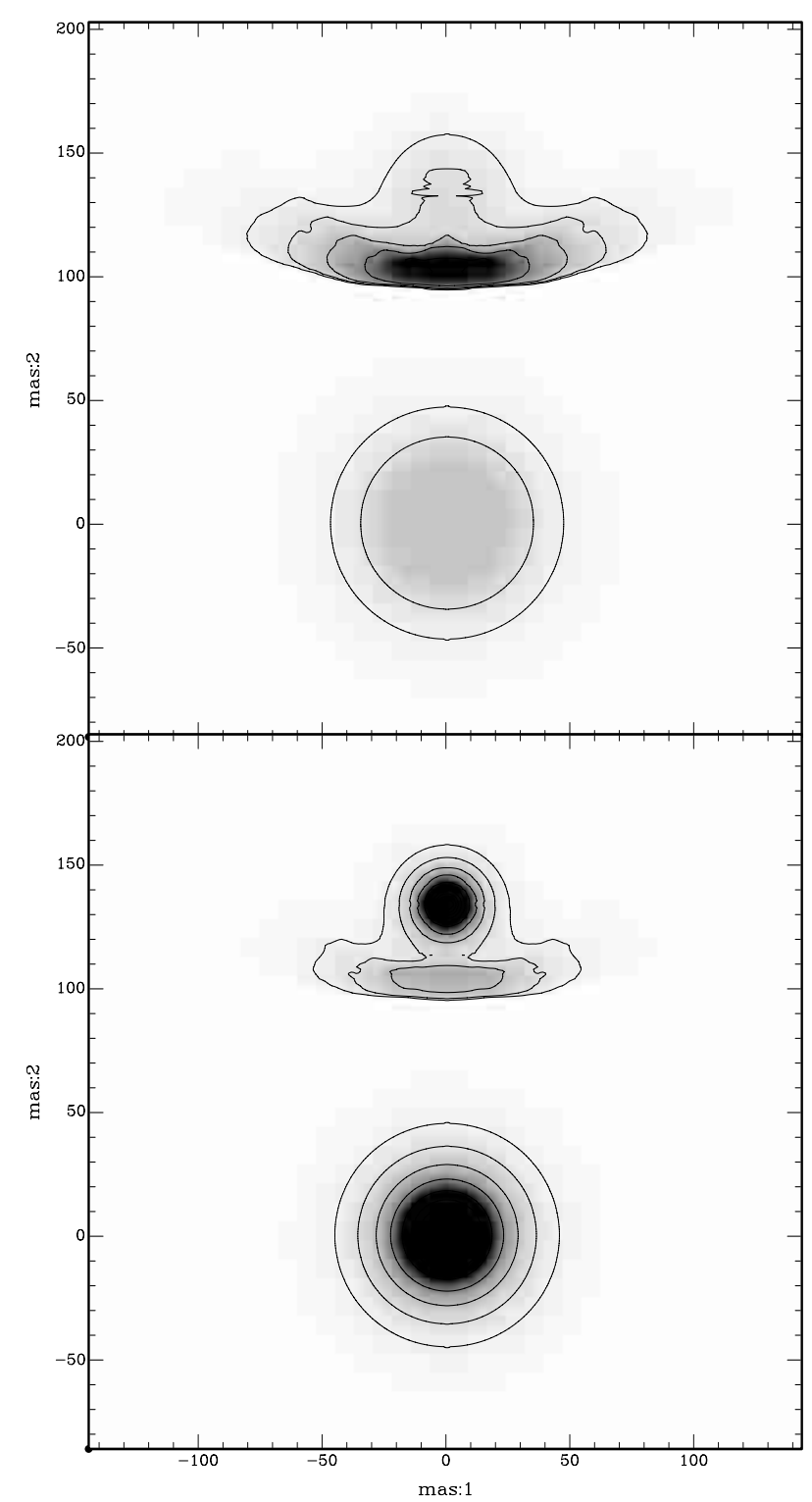

Fig. 2. Intensity distributions of our standard model at $0^{\circ}$ inclination at $1.6 \mathrm{GHz}$ (top) and $22 \mathrm{GHz}$ (bottom). Both images have the same intensity scale, highlighting the relative importance of the emission from the wind-wind collision region and the stellar winds as frequency varies (see also Fig. 17). Though not shown, the emission extends to the panel boundaries. Neither the Razin effect nor SSA are included in this calculation.

fully ionised, isothermal stellar wind envelope with a $r^{-2}$ radial density distribution.

Although we have deliberately avoided modelling a specific system in this first analysis, these initial results already bear similarities to observations e.g. WR 147 - see Sect. 4. The total flux, the spectral shape, and the spatial intensity distribution are of the correct order of magnitude and morphology.

\subsection{Razin effect and Synchrotron Self-Absorption}

In Figs. 3 and 4 the influence of the Razin effect and SSA on the synchrotron spectrum of our standard model is shown compared with the case where neither mechanism is active. 


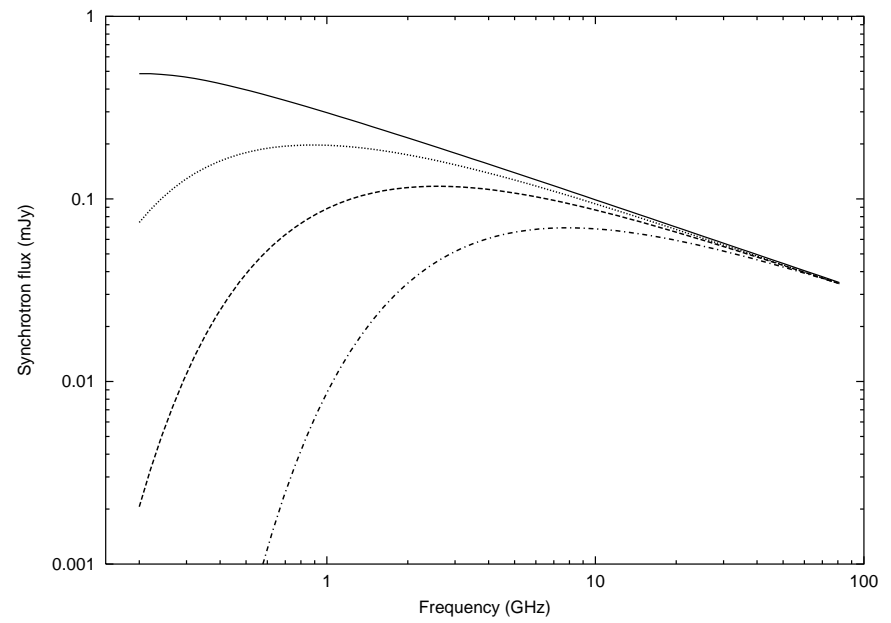

Fig. 3. The influence of the Razin effect on synthetic synchrotron spectra using the standard model at $0^{\circ}$ inclination for various values of $\zeta$. The effect of free-free absorption by the circum-binary stellar wind envelope is negligible, just visible at around 200-300 MHz. No Razin (solid), $\zeta=10^{-3}$ (dotted), $10^{-4}$ (dashed), $10^{-5}$ (dash-dotted). All spectra are normalized to the $80 \mathrm{GHz}$ synchrotron flux when no Razin effect is included.

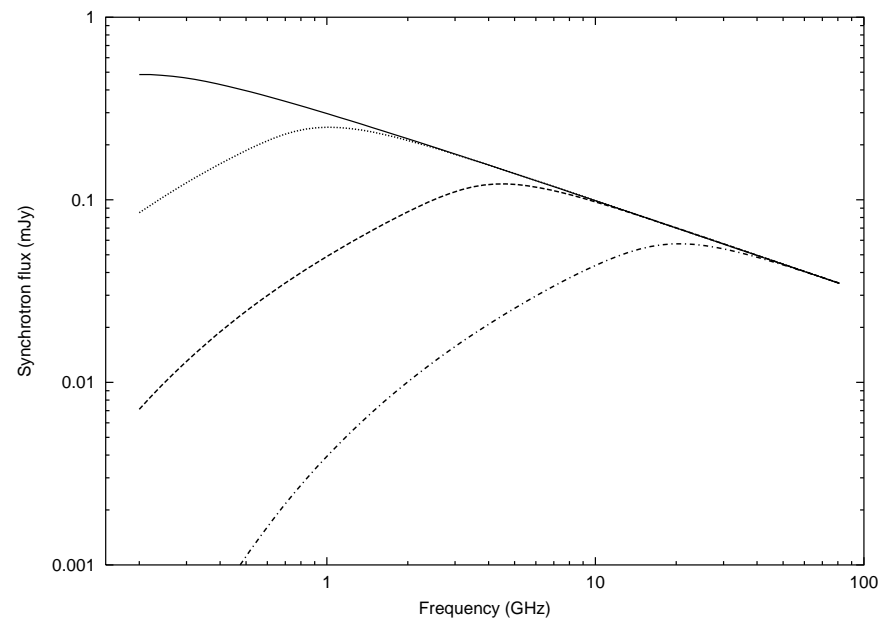

Fig. 4. The effect of SSA on synthetic synchrotron spectra using the standard model at $0^{\circ}$ inclination for various values of $\zeta$. The effect of free-free absorption by the circum-binary stellar wind envelope is negligible, just visible at around 200-300 MHz. No SSA (solid), $\zeta=10^{-4}$ (dotted), $10^{-3}$ (dashed), $10^{-2}$ (dash-dotted). All spectra are normalized to the $80 \mathrm{GHz}$ synchrotron flux when no SSA is included.

Note that in the models shown, free-free opacity is negligible and has little influence on the spectra. It is clear that both the Razin effect and SSA can be important in quenching the low-frequency spectrum, with the relative impact of these two mechanisms in these models being strongly dependent on the value of $\zeta$. For $\zeta=10^{-4}$, the Razin effect is the dominant mechanism whereas for $\zeta=10^{-3}$, SSA dominates. From Eqs. (11) and (13), $\alpha_{v} \propto \zeta^{2}$ and $\nu_{\mathrm{R}} \propto \zeta^{-\frac{1}{2}}$ (for fixed $n_{\mathrm{e}}$ ) so we expect SSA to be increasingly important as $\zeta$ increases, with the Razin effect becoming more important for decreasing $\zeta$. For our standard model and reasonable values of $\gamma_{\max }$, we find that the Razin effect dominates the low-frequency absorption when $\zeta \lesssim 10^{-4}$, the Razin effect and SSA are roughly comparable when $\zeta \sim 10^{-3}$, and SSA dominates when $\zeta \gtrsim$ $10^{-2}$. Of course, we expect these values to be different for other model systems.

For a given value of $\zeta$, a different value of $\gamma_{\max }$ would affect the value of $C$ (Eq. (9)) and hence the intrinsic synchrotron flux, $P(v)$ (Eq. (6)), although the synchrotron spectrum would still extend to infinite frequency as a result of adopting Eq. (6). To obtain a certain intrinsic flux, only the combination $\zeta^{7 / 4} / \ln \gamma_{\max }$ is required to be a specific value and $\zeta$ and $\gamma_{\max }$ can vary within this constraint. However, since $\alpha_{v}$ (Eq. (11)) and $\nu_{\mathrm{R}}$ (Eq. (13)) depend on different powers of $\zeta$ and $\gamma_{\max }$, this degeneracy breaks down when SSA and the Razin effect are important. This means that the application of our model is dependent on the particular value of $\gamma_{\max }$ assumed. Nevertheless, since $\ln \gamma_{\max }$ will vary by less than a factor of 4 for a reasonable range of $\gamma_{\max }$, the strength of these absorption terms will not change a great deal. In fact, as $\alpha_{v} \propto \zeta^{2} / \ln \gamma_{\max }$ i.e. almost the same dependence as the intrinsic flux, its variation will be very small. As $v_{\mathrm{R}} \propto \zeta^{-1 / 2}$, the relative strength of the Razin effect is slightly more sensitive to our particular choice of $\gamma_{\max }$, but not hugely so. Hence the curves in Figs. 3 and 4 are not strictly functions of only $\zeta$, as there is a slight implicit dependence on $\gamma_{\max }$ as well.

Figures 3 and 4 also demonstrate that the spectral shape belies the underlying mechanism; the SSA spectra are powerlaw, whereas the Razin dominated spectra decay exponentially. Optically thick synchrotron emission actually has a power-law spectrum with a slope of +2.5 , but we find that the spectral slope in our calculations is $\sim+1$. This difference reflects the fact that the synchrotron emission in our models is always a mixture of optically thick and thin emission. When the synchrotron emission from the shock apex is optically thick, a substantial amount of optically thin emission from the downstream flow contributes to the total non-thermal emission.

\subsection{Effect of binary separation}

The dramatic variations in the radio flux of the highly eccentric WR+OB binary WR 140 point to the significance of binary separation to the observed radio emission. In WR 140, the separation varies from $\sim 2 \mathrm{AU}$ at periastron to $\sim 28 \mathrm{AU}$ at apastron, a separation range over which different physical mechanisms determine the resulting synchrotron spectrum. In this section, we perform calculations with varying binary separation to investigate the effect on the intrinsic synchrotron luminosity, and also how separation affects the relative importance of free-free opacity, the Razin effect, and SSA on the spectrum. We also discuss the impact of IC cooling as a function of separation.

In Fig. 5 we show the effect of free-free opacity on the spectra resulting from varying the separation between $2 \times 10^{14} \mathrm{~cm}$ and $80 \times 10^{14} \mathrm{~cm}$, while the other parameters of our standard model were unchanged. The radius of optical depth unity for the WR wind is $\approx 2 \times 10^{14} \mathrm{~cm}$ at $5 \mathrm{GHz}$. The increasing importance of free-free absorption as the separation decreases is clearly observed at the lower frequencies. As the stars move closer together the wind-wind-collision region moves closer to both the WR star and the companion, and is surrounded by 


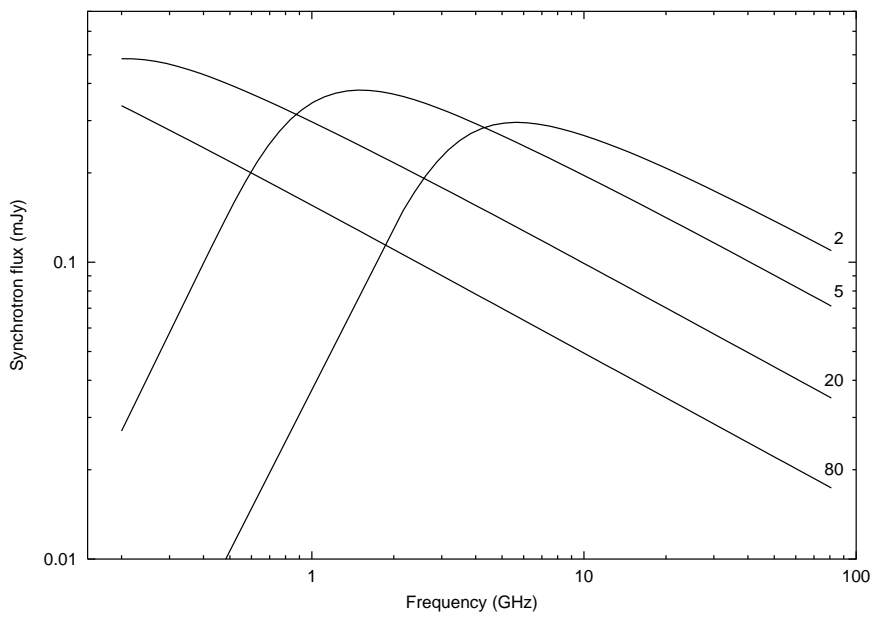

Fig. 5. The effect of separation on synchrotron spectra using the standard model at $0^{\circ}$ inclination and $\zeta=10^{-4}$, for $D=2,5,20$, and $80 \times 10^{14} \mathrm{~cm}$. Only the effect of free-free opacity is included.

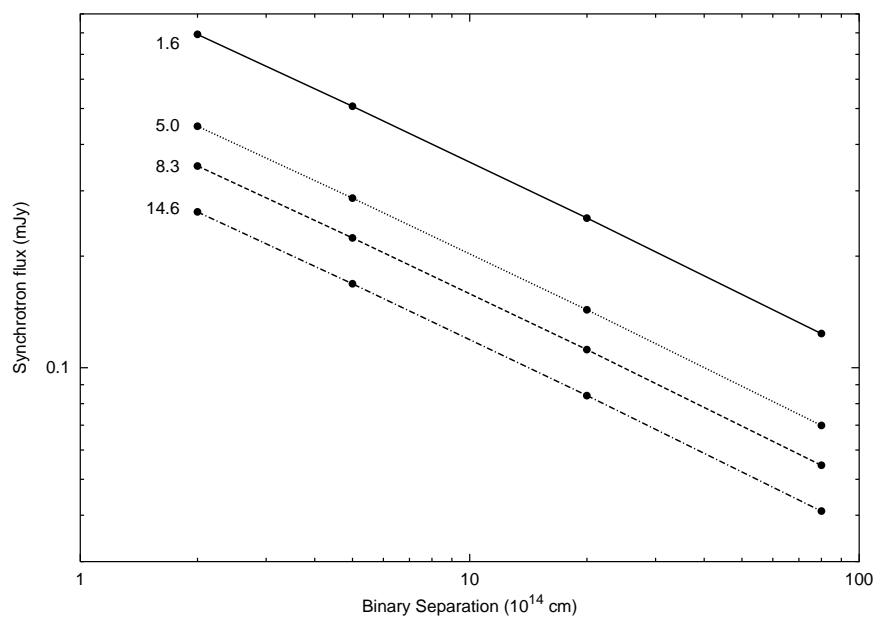

Fig. 6. The effect of separation on the synchrotron luminosity for a power-law electron energy distribution in the optically thin regime. Shown are the data points at four frequencies: 1.6 (solid), 5.0 (dotted), 8.3 (dashed), $14.6 \mathrm{GHz}$ (dot-dashed). The slope of this log-log plot is $-1 / 2$, as expected.

increasingly dense gas which increases the line-of-sight opacity to the shock apex. For a stellar wind with an $r^{-2}$ radial density distribution it can be easily shown that the line-of-sight opacity $\tau$, at frequency $v$ through the wind is $\tau \propto \xi^{-3} v^{-2.1}$, where $\xi$ is the impact parameter. Since $\xi \propto D$ for a given inclination, the turnover frequency for a constant opacity value is $v \propto D^{-10 / 7}$, in excellent agreement with the spectra in Fig. 5.

With the parameters for our standard model, Fig. 5 also reveals that the synchrotron luminosity increases as the separation decreases, due to the increased thermal energy density in the collision region. From Eq. (6), it can be deduced that the intrinsic synchrotron emission per unit volume $P(v) \propto$ $\zeta^{3 / 4} n^{3 / 4} v^{-1 / 2}$ for an electron power-law index $p=2$, in the absence of SSA or the Razin effect. Since the post-shock density $\propto D^{-2}$ and the volume of the emitting region scales as $D^{3}$, the total synchrotron emission from the entire wind collision volume $\propto D^{-1 / 2} v^{-1 / 2}$. This is illustrated in Fig. 6. For comparison, we note that the total intrinsic X-ray emission from the

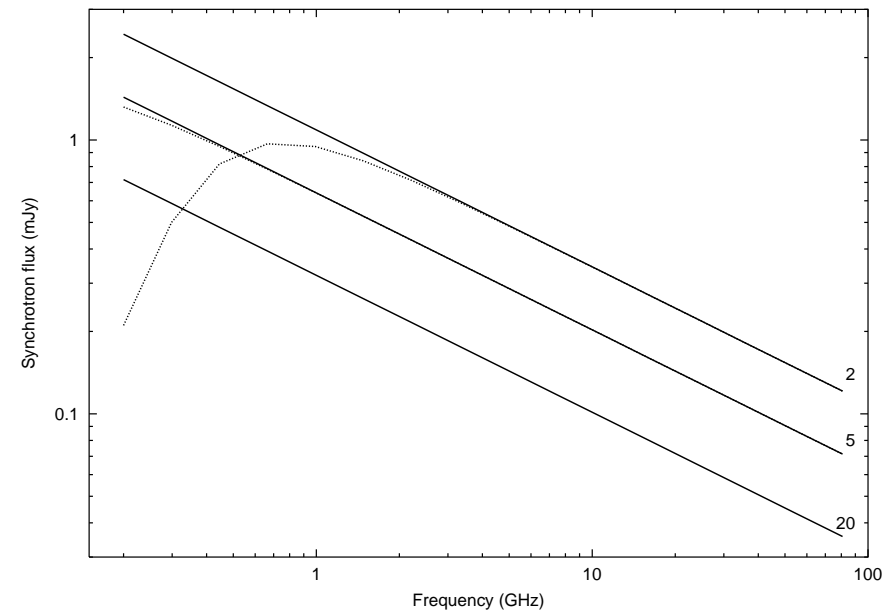

Fig. 7. The effect of separation on the free-free opacity within the wind-wind collision region, using the standard model at $0^{\circ}$ inclination and $\zeta=10^{-4}$, for $D=2,5$, and $20 \times 10^{14} \mathrm{~cm}$. The intrinsic synchrotron emission is shown (solid) along with the spectra of the synchrotron emission emerging from the collision zone (dotted).

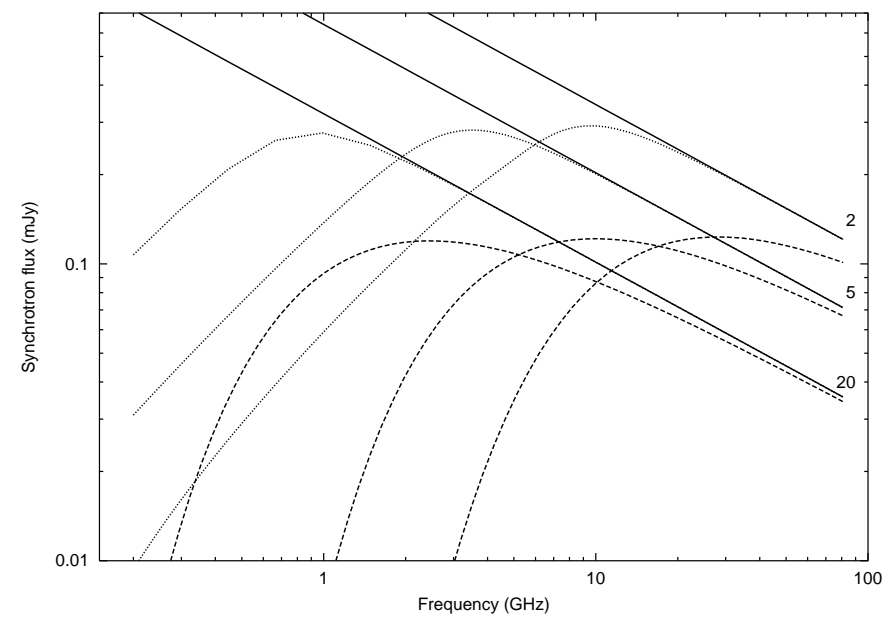

Fig. 8. The effect of separation on the synchrotron emission using the standard model at $0^{\circ}$ inclination and $\zeta=10^{-4}$, for $D=20 \times 10^{14}$ (labelled "20"), $5 \times 10^{14} \mathrm{~cm}$ ("5") and $D=2 \times 10^{14}$ ("2"): the intrinsic synchrotron emission i.e. no Razin or SSA (solid); only Razin effect included (dashed); only SSA included (dotted). The intrinsic synchrotron emission is shown to highlight the effect of separation on the synchrotron emission i.e. in the absence of the strong free-free absorption, as seen in Fig. 5.

wind-wind collision scales as $D^{-1}$ in the optically thin, adiabatic limit (Stevens et al. 1992).

In Fig. 7 the effect of free-free opacity from within the shocked gas is shown. As the binary separation is reduced the free-free opacity in the shocked region becomes increasingly important, as density increases. Note that shocked gas temperature is independent of separation. As before, the free-free spectral turnover $\propto D^{-10 / 7}$, in close agreement with the spectra shown. In this case, in systems such as WR 140 with periaston separation $\sim 3 \times 10^{13} \mathrm{~cm}$, the free-free opacity of the shocked plasma will have an impact of the spectrum below $\sim 10 \mathrm{GHz}$.

The impact of changing separation on the importance of the Razin effect and SSA is shown in Fig. 8. The intrinsic 


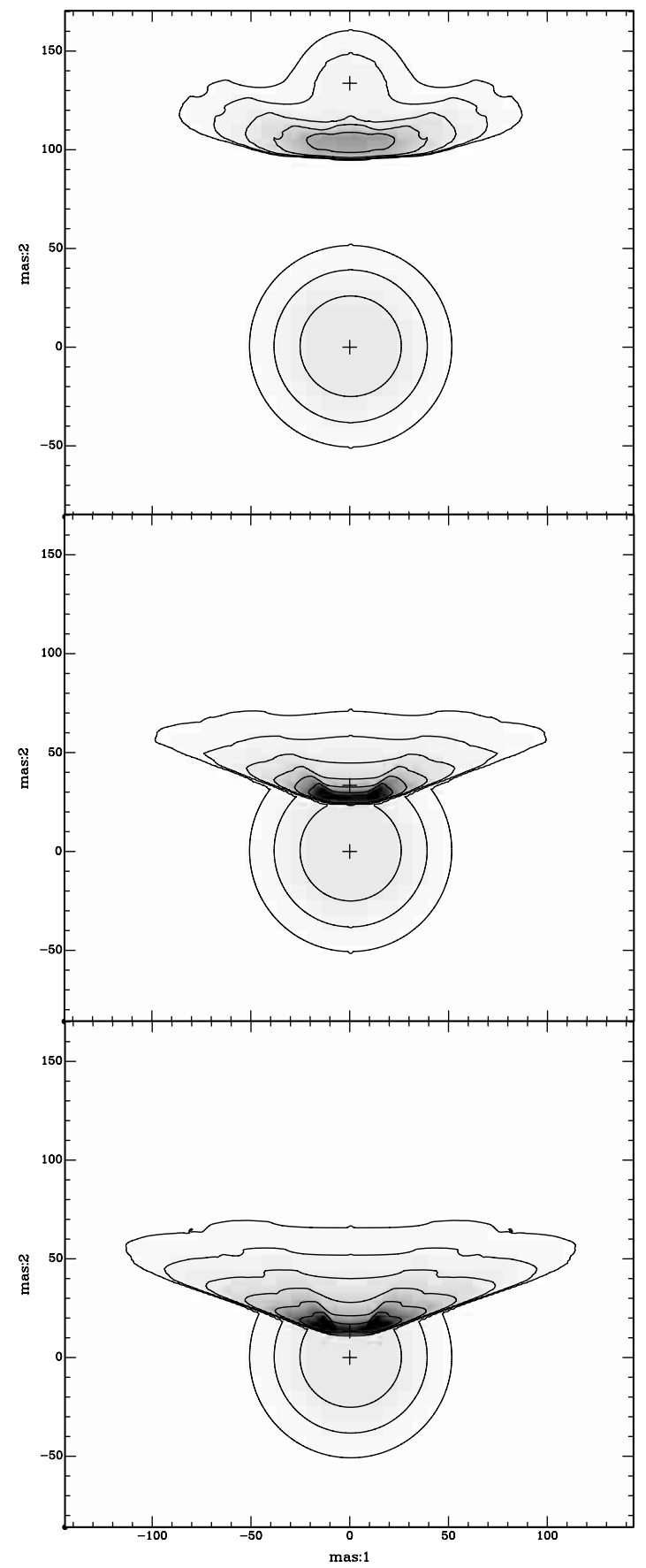

Fig. 9. Intensity distributions at $1.6 \mathrm{GHz}$ and $0^{\circ}$ inclination for an orbital separation of 20 (top), 5 (middle), and $2 \times 10^{14} \mathrm{~cm}$ (bottom), corresponding to angular separations of 133,33 and 13.3 mas respectively. The crosses denote the positions of the stars, with the WR star located at $(0,0)$. Each image has the same intensity scale and contours. The contours were chosen to highlight the relative brightness of the stellar wind of the WR star and the emission from the collision region. Though not shown, the emission extends to the boundaries of the panels (see also Fig. 17).

synchrotron spectrum is shown in order that the dramatic effects of the free-free opacity of both the unshocked circumbinary stellar winds and the shocked gas, clearly seen in Figs. 5 and 7, are removed. As expected, the attenuation from both processes is more pronounced as the separation decreases since the shock apex occurs in a higher density part of the stellar wind envelope. The Razin turnover frequency $v_{\mathrm{R}} \propto n / B$, and as $B \propto n^{\frac{1}{2}}$ (for fixed $\zeta$ ), $\nu_{\mathrm{R}}$ increases as $n^{\frac{1}{2}}$. Because $n \propto D^{-2}$, we find that $v_{\mathrm{R}} \propto D^{-1}$. With $D=2 \times 10^{15} \mathrm{~cm}$, our standard model gives $v_{\mathrm{R}}=1.3 \mathrm{GHz}$ for emission at the shock apex. Away from the shock apex $v_{\mathrm{R}}$ will be smaller. For our model with $D=5 \times 10^{14} \mathrm{~cm}, v_{\mathrm{R}}=5 \mathrm{GHz}$. Both of these values are in excellent agreement with the frequency where the Razin effect causes a $1 / e$ reduction in flux, as seen in Fig. 5. The opacity due to SSA is $\tau_{\mathrm{SSA}} \sim \alpha_{v} r_{\mathrm{OB}}$, where $r_{\mathrm{OB}}$ is the distance between the shock apex and the OB star. Since $\alpha_{v} \propto D^{-4} v^{-3}$ and $r_{\mathrm{OB}} \propto D$ (see Eq. (15)), this leads to $v_{\mathrm{SSA}} \propto D^{-1}$, which is in excellent agreement with the spectra shown in Fig. 5, where the SSA turnovers occur at approximately $0.9,3.5$, and $9.0 \mathrm{GHz}$ for separations of 2,5 and $20 \times 10^{14} \mathrm{~cm}$ respectively.

Also of interest is how the distribution of the synchrotron emission is affected by changing separation. This is shown in Fig. 9 for three different separations. In the middle and bottom images, the maximum intensity actually occurs to either side of the axis of symmetry, this offset widening as the separation decreases. This is consistent with significant absorption along the line of sight to the shock apex, and slightly reduced absorption to the positions of peak emission. In addition, a given flux intensity from the wind collision is apparent out to greater off-axis distances as the separation decreases. This is due to a combination of increased energy density in the collision zone and the importance of absorption from the surrounding winds.

As mentioned in Sect. 2.5, IC scattering is an important cooling mechanism for relativistic electrons. To estimate when IC cooling needs to be considered, we determine the minimum value of $\gamma$ at which IC cooling can have an effect before the relativistic electrons adiabatically flow out of the system. The distance from the OB companion to the shock apex is given by

$r_{\mathrm{OB}}=\frac{\eta^{\frac{1}{2}}}{1+\eta^{\frac{1}{2}}} D$.

Here, $\eta$ is the momentum ratio of the two stellar winds in the system and $D$ is the binary separation. For our standard model, $\eta=0.1$ and $r_{\mathrm{OB}} / D=0.24$. Assuming a strong shock, and that the emission is concentrated near the shock apex within an offaxis distance of $\pi r_{\mathrm{OB}} / 2^{1}$, the characteristic flow timescale for gas is $t_{\text {flow }}=2 \pi r_{\mathrm{OB}} / v$, where the post-shock velocity is $v / 4$, with $v$ being the relevant pre-shock velocity of either the WR or OB stellar wind.

For relativistic particles with $v / c \approx 1$, the rate of energy loss through IC cooling is (Rybicki \& Lightman 1979)

$$
\left.\frac{\mathrm{d} E}{\mathrm{~d} t}\right|_{\mathrm{IC}}=\frac{\sigma_{T} \gamma^{2}}{3 \pi} \frac{L_{*}}{r^{2}}
$$

1 This is true in the wider systems, but in close systems strong absorption along the line of sight to the shock apex can create flux maxima to either side of the shock apex, as shown in Fig. 9. However, in such cases a substantial proportion of the mass flux (relativistic electron flux) through these regions will have passed through (originated in) the shock at some non-negligible off-axis distance. Therefore, the following argument should be good to first order, even in close systems. 
where $L_{*}$ is the stellar luminosity and $r$ is the distance of the shock from the star. Since the relative momentum fluxes of the WR and OB stellar winds normally cause the collision zone to be closer to the OB star, which is generally also the more luminous star in the binary, $L_{*}$ and $r$ should be evaluated for the OB star. From Eq. (16), the timescale for $50 \%$ energy loss through IC scattering is

$t_{\mathrm{IC}}=\frac{3 \pi m_{\mathrm{e}} c^{2} r_{\mathrm{OB}}^{2}}{\sigma_{\mathrm{T}} L_{*} \gamma}$,

where we have assumed that to first order the average distance of the relativistic electrons from the source of UV photons during this time is $r_{\mathrm{OB}}$. If the distance from $\mathrm{OB}$ star (the dominant source of UV photons) to the relativistic electrons is such that an electron of energy $\gamma m_{\mathrm{e}} c^{2}$ loses $50 \%$ of its energy through IC cooling during the flow time, then $t_{\text {flow }}=t_{\text {ic }}$ when

$r_{\mathrm{OB}}=\frac{2 \sigma_{\mathrm{T}} L_{*} \gamma_{\text {flow }}}{3 m_{\mathrm{e}} c^{2} v}$

or alternatively, when

$\gamma_{\text {flow }}=\frac{3 m_{\mathrm{e}} c^{2} v r_{\mathrm{OB}}}{2 \sigma_{\mathrm{T}} L_{*}}$.

If we assume further that the luminosity of the $\mathrm{OB}$ star is $L_{*}=10^{5} L_{\odot}$, then from Eq. (19) we find $\gamma \approx 500$ for our standard model, which from Eq. (10) gives a characteristic frequency $v_{\mathrm{c}} \approx 6 \mathrm{GHz}$ for $B=6 \mathrm{mG}$. Hence, for our standard model, the above order of magnitude estimate suggests that IC cooling starts to have an effect on the relativistic electrons with $\gamma>\gamma_{\text {flow }}$. In practice, this is likely to represent a lower limit. Firstly, it has been derived for electrons accelerated at the symmetry axis and which then flow to a distance of $\pi r_{\mathrm{OB}} / 2$ from the symmetry axis. Electrons accelerated somewhat offaxis will, of course, take less time than $t_{\text {flow }}$ to reach this off-axis distance. Moreover, the average distance to the OB star will be greater than $r_{\mathrm{OB}}$. Therefore, we expect that IC cooling has only a significant effect for $\gamma$ greater than a few times $\gamma_{\text {flow }}$. For our standard model, electrons with $\gamma \sim 3 \gamma_{\text {flow }}$ result in emission with a characteristic frequency of $\sim 50 \mathrm{GHz}$.

This estimate suggests that IC cooling is an important consideration in all systems, regardless of binary separation, for sufficiently high frequencies (or $\gamma$ ). However, it is clearly most significant in shorter period systems, since $\gamma_{\text {flow }} \propto D$ and $v_{\mathrm{c}} \propto \gamma^{2} B \propto D$ (assuming $\left.B \propto 1 / D\right)$. Models with IC cooling included explicitly will be investigated in Pittard et al. (in preparation).

\subsection{Effect of inclination angle}

We now examine the effect on the observable synchrotron spectrum of changing the inclination of the axis of symmetry (the line of centres) to the line of sight using our standard model. This is shown in Fig. 10, where only the effect of the freefree opacity of the circum-binary stellar wind envelope is being considered. Since our model is axis-symmetric, changing only the inclination covers any orientation of the system relative to the observer. The largest effects are seen at the lowest

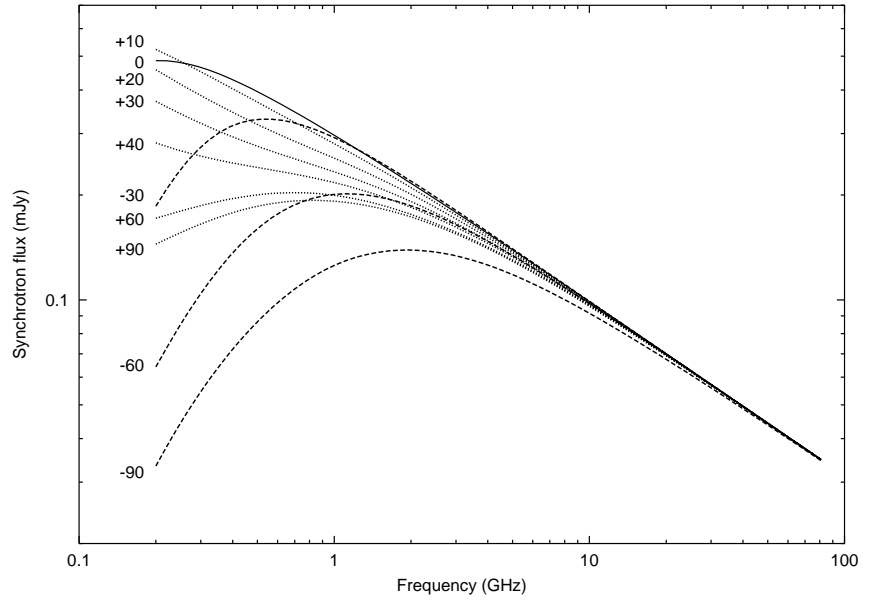

Fig. 10. The effect of free-free opacity as a function of inclination angle on the synchrotron spectra of the standard model with $\zeta=10^{-4}$. The inclination angle of the axis of symmetry to the plane of the sky for the various spectra is shown. Dashed lines indicate $i<0^{\circ}$ (i.e. WR star in front), the solid line $i=0^{\circ}$ (i.e. quadrature), and dotted lines $i>0^{\circ}$ (i.e. OB star in front).

frequencies, where the free-free opacity is highest for a given path length through the circum-binary envelope $\left(\tau_{\mathrm{ff}} \propto v^{-2.1}\right)$. At an inclination angle $i=0^{\circ}$, the lines-of-sight are perpendicular to the axis of symmetry of the model; at $i=-90^{\circ}$ they pass first through the WR-star wind; and at $i=+90^{\circ}$ they pass first through the OB-star wind. The asymptotic half-opening angle of the WR-shock cone is $72^{\circ}$, so when $i>18^{\circ}$, lines of sight into the system can impinge the shocked region without first passing through unshocked circum-binary gas.

The effects of changing free-free opacity with inclination are clear. The size of the optically thick region of the circumbinary nebula goes as $v^{-0.7}$ for an $r^{-2}$ radial density gradient, and so as frequency increases the opacity is reduced along any given line-of-sight. In our standard model, it is evident that the cirumbinary nebula is optically thin above a few GHz. The largest free-free attenuation occurs when the line of sight to the wind-wind collision region passes closest to the WR star i.e. at $i=-90^{\circ}$. As the inclination angle increases, the line of sight passes through less dense regions of the WR star and the opacity is reduced, resulting in higher observed synchrotron flux. For $i=0^{\circ}$ the lines of sight to the wind-wind collision region pass largely through the outer regions of the WR star envelope and the low free-free opacity of the wind-wind collision region. For $i>0^{\circ}$, the region of the wind-wind collision zone occulted by the optically thick surface of the OB-star wind moves gradually toward the shock apex as $i$ increases (cf. Fig. 11). This, and the fact that the lines of sight to the shock apex pass through the dense inner regions of the OB-star wind, is the cause of the increasing absorption at low frequencies with increasing $i$. The flux when $i=+90^{\circ}$ is always greater than the flux when $i=-90^{\circ}$ since the optical depth unity surface of the WR wind is always larger than that of the OB-star wind owing to the higher density of the WR wind.

The effect of inclination on the resulting intensity distribution is demonstrated in Fig. 11, where the line-of-sight passes down the shock cone, and the OB-star wind absorbs flux from 


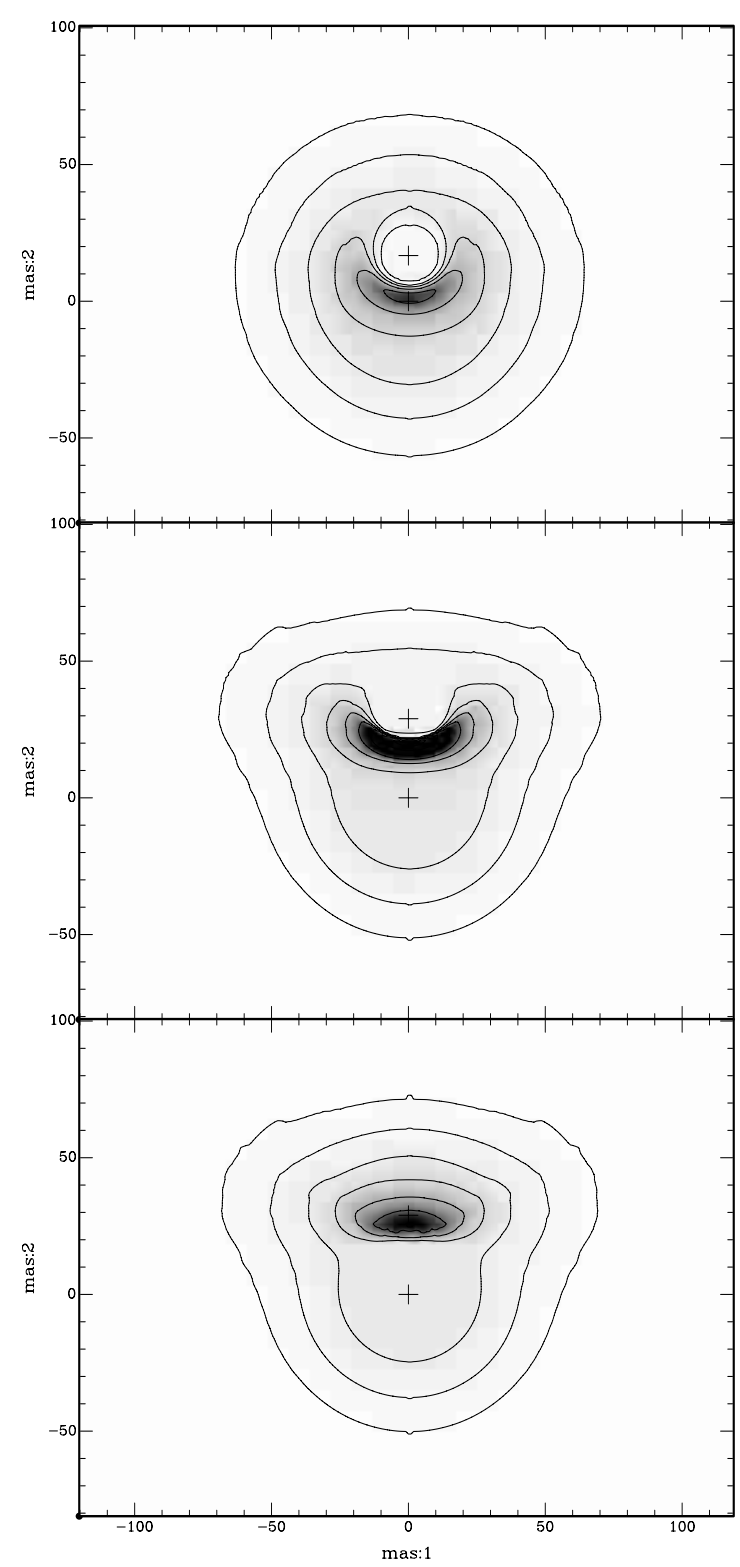

Fig. 11. The 1.6-GHz intensity distribution for a model with $D=$ $5 \times 10^{14} \mathrm{~cm}$ at $+60^{\circ}$ (top), $+30^{\circ}$ (middle), and $-30^{\circ}$ (bottom) inclination. At positive angles the $\mathrm{OB}$ star is closer to the observer than the WR star. The crosses denote the position of the stars, with the WR star at $(0,0)$. Each image has the same intensity greyscale and contour intervals (also used in the middle panel of Fig. 9, showing the $0^{\circ}$ case). The optically thick part of the OB-star wind is the obvious "hole" (top panel), below which the synchrotron emission is seen, surrounded by the free-free emission, largely from the WR wind. As inclination decreases, the hole becomes less pronounced and the synchrotron intensity increases as the line-of-sight opacity through the OB wind decreases. When the WR star is closer to the observer $(i<0)$, the optically thick part of the WR-star wind obscures the lower part of synchrotron emission region.

its far side. Absorption by the optically thick region of the OB-star wind is distinctly apparent. Clearly the inclination angle of a given source can have a large effect on the resulting flux and the spatial intensity distribution.

The Razin effect only affects the generation of synchrotron photons and is independent of inclination. On the other hand,

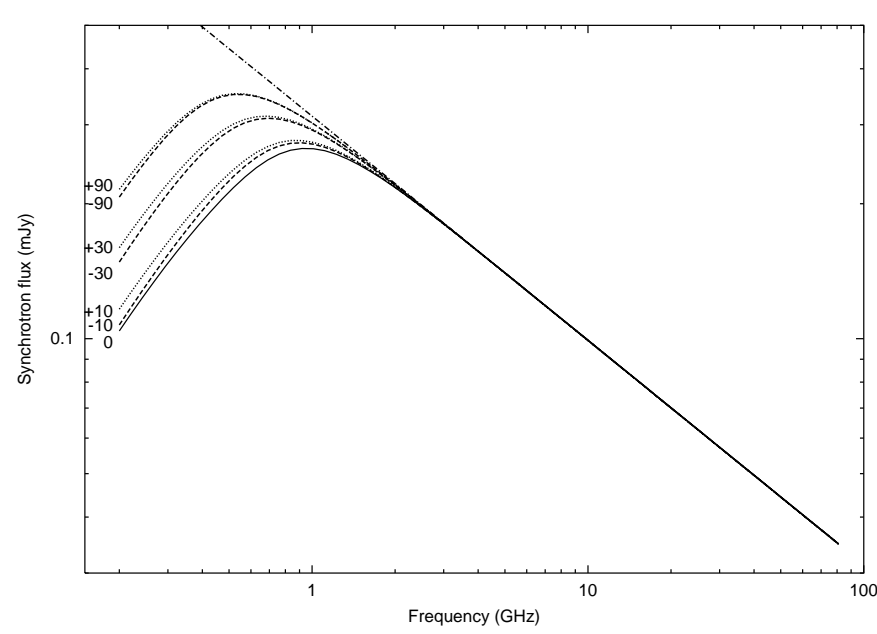

Fig. 12. The effect of SSA on the spectrum of the emission emerging from the shocked region as a function of inclination angle for the standard model with $\zeta=10^{-4}$. The inclination angle of the axis of symmetry to the plane of the sky for the various spectra is shown. Dashed lines indicate $i<0^{\circ}$ (i.e. WR star in front), the solid line $i=0^{\circ}$ (i.e. quadrature), and dotted lines $i>0^{\circ}$ (i.e. OB star in front). The dot-dashed line is the intrinsic synchrotron emission from the shocked gas, which is independent of inclination, plotted for reference.

SSA is dependent on the path length through the shocked gas, and inclination angle influences its effect on the low frequency synchrotron spectrum. This is shown in Fig. 12, where the intrinsic synchrotron spectrum is shown. Examining the intrinsic spectrum eliminates the effect of the large changes in free-free opacity with inclination angle, as seen in Fig. 10. The dependence of SSA on the inclination angle is clearly a function of the path length through the shocked envelope, particularly the highest density (shock apex region) part of the shocked gas. Thus at high angles, this path length is small and the impact of SSA on the spectrum is lowest, whereas at quadrature the lines-of-sight through the shock apex region are at maximum length and the impact of SSA on the spectrum is highest. However, the effect is not as dramatic a function of inclination angle as the effect of varying free-free opacity with inclination angle.

When the Razin effect, SSA, and free-free opacity are considered together in our models it is important to bear in mind that both the Razin and SSA are functions of $\zeta$ (see Sect. 3.2). As an example, when $\zeta=10^{-4}$, the Razin effect is so dominant that the spectrum is influenced little by changes in the inclination angle. The changes that do occur are due mostly to the changing free-free opacity.

In Fig. 13 we show how the thermal component varies with inclination angle in our standard model. The observed free-free flux is essentially constant throughout the range of inclinations, except when $i$ approaches $-90^{\circ}$ and $+90^{\circ}$. At these extreme inclinations, the optically thick parts of, respectively, the WR and $\mathrm{OB}$ star winds absorb the free-free emission arising from the bulk of the stellar wind of the other star and from the windwind collision region (a relatively small contribution to the total thermal emission (Stevens 1995)), producing a decrease in thermal flux. The free-free flux at $i=-90^{\circ}$ is slightly higher than 


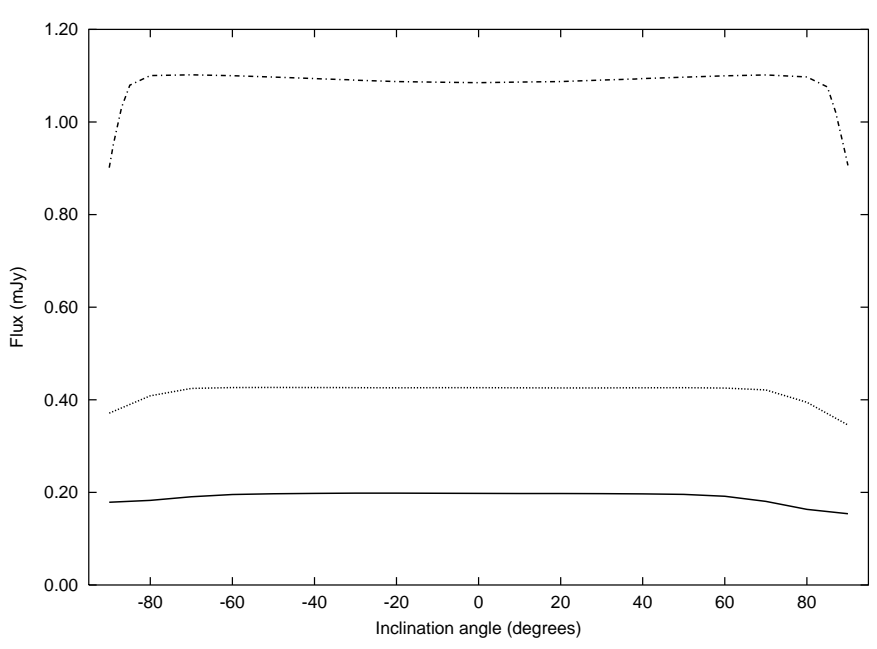

Fig. 13. The change in thermal flux as a function of inclination angle using the standard model with $\zeta=10^{-4}$. Shown are curves for 1.6 (solid), 5 (dotted) and $22 \mathrm{GHz}$ (dot-dashed).

at $i=+90^{\circ}$, consistent with the higher density of the WR star wind.

\section{Modelling the radio emission from WR 147}

Having explored how the radio flux varies with separation and inclination in the previous section, we now turn our attention to the modelling of a specific system. WR 147 is notable for being among the brightest WR stars at radio frequencies, and for being one of two systems in which the thermal and synchrotron emission are observed to arise from two spatially resolved regions e.g. Williams et al. (1997, and references therein). Furthermore, it is one of a handful of WR+OB binary systems where the two stars are spatially resolved (Williams et al. 1997; Niemela et al. 1998). These observations suggest a very wide system with a projected separation $D \cos i=0.635 \pm 0.020^{\prime \prime}$. At the estimated distance of $\sim 0.65 \mathrm{kpc}$ (Churchwell et al. 1992; Morris et al. 2000) this corresponds to a separation $D \sim 415 / \cos i$ AU. This relationship between $D$ and $i$ represents an important constraint for any models of the system. The inclination angle is unknown so we investigate models for several different values which requires different values of the physical separation, $D$, to maintain the observed angular separation. This leads to different sets of physical parameters, including the normalisation constant $\zeta$, to fit the observed spectrum.

\subsection{Modelling the radio spectrum of WR 147}

The radio spectrum of WR 147 is perhaps the best observed of all massive binary systems, with radiometry extending from $353 \mathrm{MHz}$ to $42.9 \mathrm{GHz}$. Reviewing the literature, it is immediately apparent that differences in excess of $50 \%$ exist in the derived synchrotron flux at some frequencies (cf. Fig. 14), giving rise to uncertainty in the position of the low frequency turnover. Skinner et al. (1999) estimate that at $5 \mathrm{GHz}$ the synchrotron flux $\left(S_{5 \mathrm{GHz}}\right)$ is greater than the synchrotron flux at $1.4 \mathrm{GHz}$, whereas Setia Gunawan et al. (2001) estimate that $S_{1.4 \mathrm{GHz}}>S_{5 \mathrm{GHz}}$. These differences may be attributed to

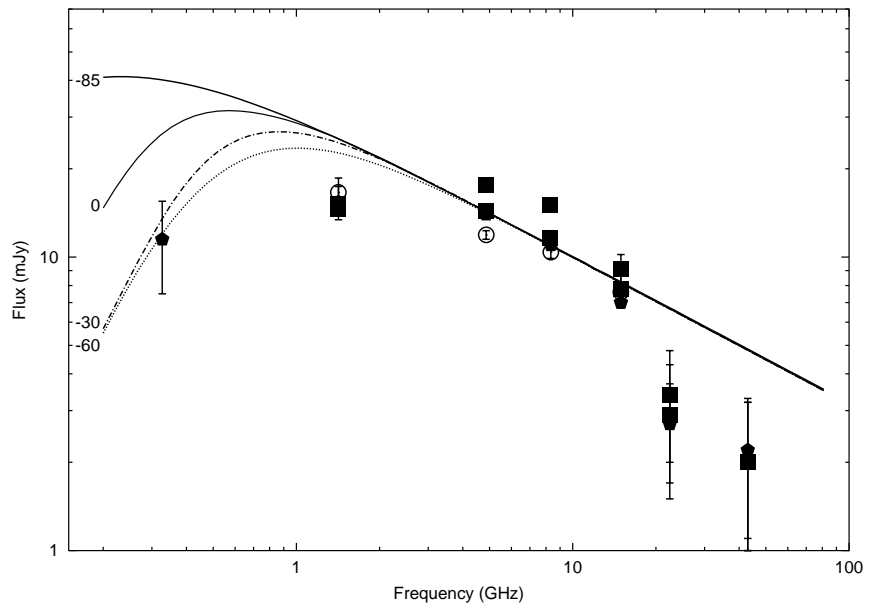

Fig. 14. The synchrotron spectrum of WR 147 as deduced by several authors - solid squares (two separate estimates from the same observational data by Skinner et al. 1999), solid pentagons (Setia Gunawan et al. 2001), open circles (fluxes from Churchwell et al. 1992 and Contreras \& Rodríguez 1999). The flux at both 22 and $43 \mathrm{GHz}$ is highly uncertain, with the latter estimated from a long extrapolation of a power-law fit to the lower frequency "thermal" data points to be $\approx 2$ mJy (Skinner et al. 1999; Setia Gunawan et al. 2001). Both high frequency points suggest a high frequency turn down. The lines are theoretical spectra from models where only free-free absorption in the circum-binary stellar wind envelope is attenuating the synchrotron emission. Models for various inclination angles are shown: $0,-30$, -60 and $-85^{\circ}$ (corresponding to $\zeta=6.92,7.23,8.08$, and $13.44 \times 10^{-3}$ respectively). The $0^{\circ}$ model is denoted by the solid line.

a number of factors that include analysis techniques, resolution effects, the assumed thermal contribution, and possibly temporal variations. Though Churchwell et al. (1992) report variations in radio flux approaching 50\%, subsequent reanalysis of the same data by Setia Gunawan et al. (2001) suggests that any variations are much smaller $(\sim 15 \%)$ and are long-term, with little power on time scales of days. Skinner et al. (1999) completed their observations of WR 147 within a couple of days, and their repeated observation at $1.4 \mathrm{GHz}$ showed no variation. This suggests that, at the very least, their observed spectrum is free of temporal variations. In addition, it is clear there are difficulties in determining the component fluxes when the sources are spatially resolved cf. Table 1 in Skinner et al. (1999) and Table 3 in Churchwell et al. (1992).

These differences form the fundamental reason for conflicting conclusions about the nature of the underlying electron energy spectrum in the current literature. While both Skinner et al. (1999) and Setia Gunawan et al. (2001) note that a freefree absorbed synchrotron power-law model is a poor fit to the data, Setia Gunawan et al. (2001) argue that the deficit in the observed flux above $15 \mathrm{GHz}$ is due to a high energy limit to the electron acceleration. In contrast, Skinner et al. (1999) argue that the spectrum is best fit by a mono-energetic relativistic electron distribution. At high frequencies, the spectrum from such a distribution falls off as $P(v) \propto v^{1 / 2} \mathrm{e}^{-v}$. This fits the 15 and $22 \mathrm{GHz}$ data very well, but the flux at these frequencies are most uncertain. Furthermore, the fit of a mono-energetic electron distribution spectrum to the high frequency fluxes depends 
also on the position of the low frequency turnover which, as already noted, is uncertain. How such an electron distribution results from a shock acceleration is unclear.

Using our model, we attempt to model the spectrum of WR 147 assuming a power-law electron energy distribution. For the stellar wind parameters we adopt $\dot{M}_{\mathrm{WN} 8}=2 \times$ $10^{-5} M_{\odot} \mathrm{yr}^{-1}, v_{\infty \mathrm{WN} 8}=950 \mathrm{~km} \mathrm{~s}^{-1}, v_{\infty \mathrm{OB}}=1000 \mathrm{~km} \mathrm{~s}^{-1}$ and a wind momentum ratio $\eta=0.02$ (Pittard et al. 2002). The last is toward the higher end of reasonable estimates, and since it gives $\dot{M}_{\mathrm{OB}}=3.8 \times 10^{-7} M_{\odot} \mathrm{yr}^{-1}$ favours a companion of spectral type around late-O or early-B supergiant. Such parameters are consistent with the estimated B0.5 by Williams et al. (1997) based on an uncertain luminosity estimate, and the more recent O5-7I deduced from HST STIS spectroscopy (Lépine et al. 2001). The composition of the WN8 stellar wind is taken from Morris et al. (2000), giving $X=0.09, Y=0.89$, and $Z=0.016$. The wind temperature for both stellar winds was assumed to be $10 \mathrm{kK}$, and the dominant ionization states were $\mathrm{H}^{+}, \mathrm{He}^{+}$and $\mathrm{CNO}^{2+}$. The thermal flux is only weakly dependent on the assumed wind temperature (through the Gaunt factor), if the ionization state is fixed.

Values of $\zeta$ are estimated for each of our models by matching the model to the mean of the "observed" synchrotron $4.86 \mathrm{GHz}$ fluxes, which we take to be $14.1 \pm 0.3 \mathrm{mJy}$. We then determine the spectrum between 0.2 and $80 \mathrm{GHz}$ assuming this constant value of $\zeta$. As the inclination of the model increases, the binary separation $D$ has to increase in order to satisfy the observed constraint on $D \cos i$, forcing the need to increase $\zeta$ to maintain the 5 to $15 \mathrm{GHz}$ flux level.

To fit the thermal flux with our chosen value of $\dot{M}$ we require the winds to be clumped. This has some precedent: Morris et al. (2000) note that the observed thermal flux implies that $\dot{M} \sim 7.5 \times 10^{-5} M_{\odot} \mathrm{yr}^{-1}$ if the wind is assumed non-clumpy, but find that models with a homogeneous wind cannot match the IR helium-line profiles observed with ISO. Their derived volume-filling factor of $f \sim 0.1$ implies an actual mass-loss rate $\dot{M} \sim 2.4 \times 10^{-5} M_{\odot} \mathrm{yr}^{-1}$. We have therefore multiplied $\varepsilon_{v}^{\mathrm{ff}}$ and $\alpha_{v}^{\mathrm{ff}}$ from Eqs. (1) and (2) by a factor $1 / f$ to simulate clumping in the cool stellar winds. We assume that both the WR and O-star winds are clumped by the same factor. Also, it is assumed that the clumps are destroyed as they pass through the shocks into the wind collision zone, and therefore do not increase the free-free emission from this region. We adopt $f=0.134^{2}$ to ensure that the thermal flux at $22 \mathrm{GHz}$ remains less than the total flux.

Figure 14 demonstrates that the low-frequency turnover could potentially be accounted for in our models by free-free opacity of the circum-binary stellar wind at intermediate inclination angles, with the line of sight into the system through the

\footnotetext{
${ }^{2}$ Interestingly, this implies that the mass-loss rate of the equivalent homogeneous wind is $\dot{M}=\dot{M}_{\text {actual }} / \sqrt{f}=5.5 \times 10^{-5} M_{\odot} \mathrm{yr}^{-1}$. While this is below the observationally deduced value using a single star model, it is consistent with the 13-38\% enhancement in $S_{v}$ expected from a binary with a wind momentum ratio $\eta=0.1$ (Stevens 1995).
}

WR wind ${ }^{3}$, but not at $0^{\circ}$ or inclinations angles for which the lines-of-sight to the shock apex pass through the densest parts of the WR wind i.e. $-85^{\circ}$. The models do not fit the $1.4 \mathrm{GHz}$ fluxes particularly well. Setia Gunawan et al. (2001) arrived at a different conclusion: that free-free absorption alone was sufficient to account for the turnover, based on results from a synchrotron point-source model. We attribute our different conclusion to the extended nature of the region of shocked material in our model; although the line-of-sight to the shock apex may be optically thick, lines-of-sight to the outer regions of the windwind-collision region may remain optically thin. Maximum free-free absorption of the low frequency synchrotron emission occurs around inclination angles of $-60^{\circ}$. For still lower inclination angles, the observed flux is higher despite increased attenuation to the shock apex. This is because increased $\zeta$ leads to synchrotron emission further from the shock apex. The only manner in which free-free opacity alone could produce a sufficiently strong low-frequency turnover would be if the size of the synchrotron emission region were reduced, such that the greater attenuation near the shock apex became more significant to the observable emission. Such a reduction in the size of the emission region could be mimicked in an ad hoc fashion in our model by evolving $\zeta$ away from the shock apex. Certainly, as the shocks become more oblique moving away from the shock apex, the particle injection and acceleration efficiency is lowered (Ellison et al. 1995, 1996). The energy spectrum is also evolving since IC cooling is ongoing as the electrons advect away, though this predominantly effects the higher energy electrons, and coulombic cooling may be more important for the lower energy electrons.

Models that include the influence of both SSA and the Razin effect in addition to free-free absorption are more successful in fitting the low-frequency data. In Sect. 3, both these effects were shown to be significant, depending on the value of $\zeta$, and it seems unrealistic to exclude such fundamental processes from our models. The resulting spectra are shown in Fig. 15. As before, values of $\zeta$ have been chosen for the different models based on matching the $5-\mathrm{GHz}$ data point, and are $\sim 1 \%$, similar to the values used in models of SNR (cf. Mioduszewski et al. 2001) and of an order of magnitude expected for shock-accelerated electrons (see Sect. 2.2). As in the case of free-free opacity alone, as the separation increases, $\zeta$ increases and the amount of emission arising far from the shock apex increases. Thus the low-frequency turnover from optically thin to optically thick emission moves to lower frequencies with increasing separation. The difference between the spectra for positive and negative inclinations of the same absolute value is due to the different free-free opacity through the OB-star wind as opposed to the WR-star wind. The values of density, equipartition field, and peak temperature near the shock apex for the models shown in Fig. 15 are given in Table 1.

In Fig. 16 the resulting total, synchrotron and thermal spectra from one of our models $(i=0$, and including SSA, the Razin effect and free-free absorption) is shown against the

\footnotetext{
3 Dougherty et al. (2003) erroneously report a fit to the lowfrequency spectrum of WR 147 using only free-free absorption. Their fit was attained using SSA, in addition to free-free absorption.
} 


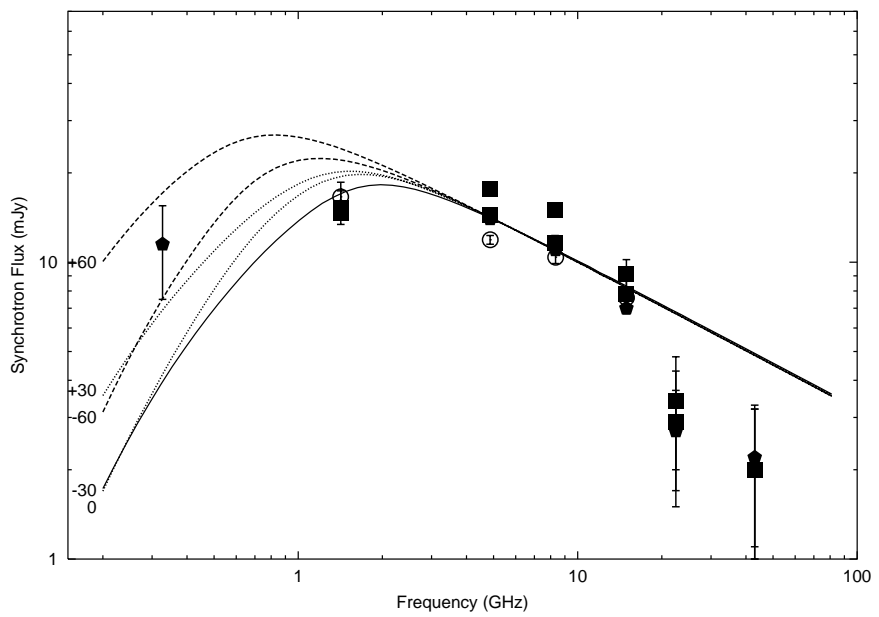

Fig. 15. Model synchrotron spectra when SSA, the Razin effect and free-free absorption in the circum-binary stellar wind envelope are included, for various inclination angles: 0 (solid), \pm 30 (dotted), and \pm 60 (dashed), with corresponding values of $\zeta=(7.03,7.32,8.12) \times 10^{-3}$. The data points are those shown in Fig. 14.

Table 1. Values of density, equipartition field, and peak temperature near the shock apex for the models shown in Fig. 15.

\begin{tabular}{llll}
\hline \hline Inclination & $0^{\circ}$ & $30^{\circ}$ & $60^{\circ}$ \\
\hline$D \times 10^{-15} \mathrm{~cm}$ & 6.21 & 7.17 & 12.4 \\
$n_{\mathrm{e}}(\mathrm{WR}) \times 10^{-4} \mathrm{~cm}^{-3}$ & 1.8 & 1.35 & 0.45 \\
$n_{\mathrm{e}}(\mathrm{OB}) \times 10^{-4} \mathrm{~cm}^{-3}$ & 3.3 & 2.5 & 0.83 \\
$B^{1} \mathrm{mG}$ & 4.1 & 3.6 & 2.2 \\
$\zeta \times 10^{3}$ & 7.03 & 7.32 & 8.12 \\
\hline$T_{\max }(\mathrm{WR}) \mathrm{K}$ & \multicolumn{3}{c}{$2.4 \times 10^{7}$} \\
$T_{\max }(\mathrm{OB}) \mathrm{K}$ & \multicolumn{3}{c}{$1.4 \times 10^{7}$} \\
\hline
\end{tabular}

${ }^{1}$ The $B$-field at the shock apex is approximately the same in both the shocked WR and OB regions.

observed spectra. Though this is not the best fit to the data in a formal sense, our model fits the data reasonably well given the approximations and assumptions which it contains. In the model shown in Fig. 16, the total flux in the 5 to $8 \mathrm{GHz}$ range is underestimated, and the synchrotron emission does not turn down somewhere around 10 to $20 \mathrm{GHz}$ to account for the observed high frequency data. We could account for the shortfall in the $5-8 \mathrm{GHz}$ total flux by increasing the thermal emission by a few mJy. This could be achieved by simply increasing $\dot{M} / v_{\infty}$ in the stellar winds. However, this would require that the synchrotron emission at frequencies higher than $\sim 10 \mathrm{GHz}$ be lower than in the current models, which may well be the case if IC cooling of the highest energy electrons is taken into account, as explained below.

Figure 16 lends considerable support to the suggestion that WR stars with continuum spectra of spectral index less than +0.6 are synchrotron emitters e.g. Dougherty \& Williams (2000). The total flux spectrum as shown in Fig. 16 could be fit with a power-law spectrum of spectral index of $\sim+0.3$, which could be interpreted as arising from a partially optically thick

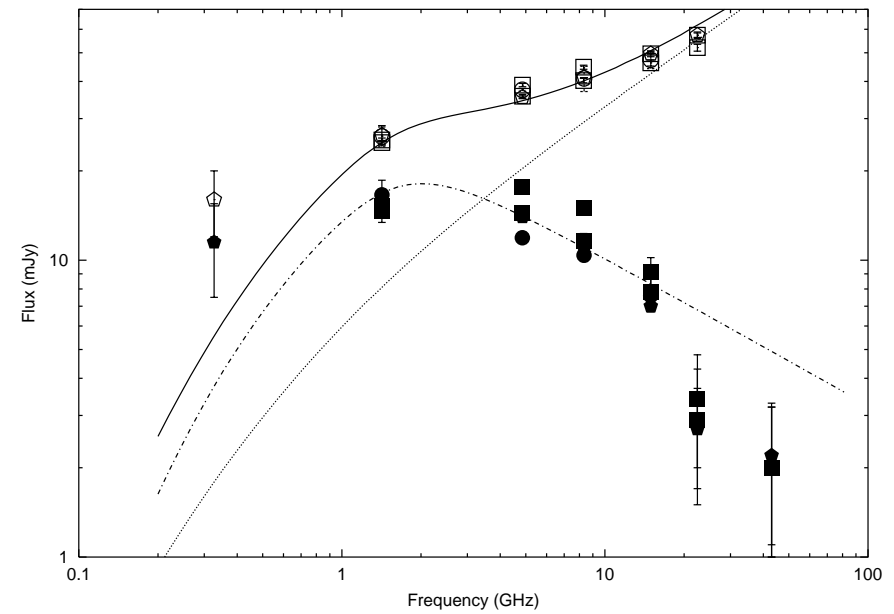

Fig. 16. The spectrum of WR 147. The synchrotron data are those shown in Figs. 14 and 15 and represented by solid data points. The total flux as deduced by the same authors are represented by the hollow data points. The lines are the total (solid), thermal (dotted) and synchrotron (dot-dashed) spectra of the $i=0^{\circ}$ models with $\zeta=7.03 \times 10^{-3}$, where SSA, Razin and free-free absorption are included in the radiative transfer calculations. The volume-filling factor $f=0.134$. Models at $+30^{\circ}$ and $-30^{\circ}$ are also consistent with the data.

stellar wind. However, it is well-established that the stellar winds of WR stars have radio continuum spectra with spectral indices $+0.6 \rightarrow+0.8$ e.g. Williams et al. (1990b); Leitherer $\&$ Robert (1991), and spectral indices less than $\sim+0.6$ are not expected from stellar winds alone. This suggests that spectral indices less than +0.6 are a strong indicator of the presence of a synchrotron component. This is especially useful in those cases e.g. WR 86, where the synchrotron and thermal emission regions are not resolved as separate components, yet a continuum spectrum with a low spectral index can be observed.

We are unwilling to draw much of a conclusion related to inclination angle from these models of the spectrum of WR 147. For models with wider separations i.e. higher inclinations, the $1.4 \mathrm{GHz}$ data is overestimated substantially, which hints at an inclination angle somewhere between $0^{\circ}$ and $30-40^{\circ}$. The high end of this estimate is consistent with the poorly constrained inclination estimates from Williams et al. (1997) and Contreras \& Rodríguez (1999). However, any conclusion related to inclination angle drawn from fitting the spectrum has to be tempered due to the heavy reliance on the accuracy of the $1.4 \mathrm{GHz}$ data points, and the poor precision of the $352 \mathrm{MHz}$ data point.

Another striking feature of Figs. $14-16$ is our inability to generate a high-frequency turnover somewhere around 10 to $20 \mathrm{GHz}$, as suggested by the data points. This is a consequence of using Eq. (6) to describe the synchrotron power, where it is assumed that $\gamma_{\max }$ is infinite. IC cooling first determines the maximum energy that can be achieved by the acceleration processes. As the relativistic electrons are advected away from the acceleration site by the flow, the highest energy particles suffer crippling losses from IC cooling as this is no longer balanced by mechanisms supplying rapid energy gain. 
Hence $\gamma_{\max }$ will have a finite value. For WR 147, the rate of energy gain by 1 st-order Fermi acceleration is equal to the rate of energy loss by IC cooling when $\gamma=\gamma_{\max } \sim 10^{6}$. From Eq. (16), electrons with $\gamma=10^{6}$ are cooled to $\gamma_{\text {flow }} \sim 400$ within a time $t_{\text {flow }}$ when $r \approx r_{\mathrm{OB}}$ and $L_{*}=10^{5} L_{\odot}$. The high frequency turn down seen in the observational data occurs at $\sim 10-20 \mathrm{GHz}$, which for $B \sim 3 \mathrm{mG}$ suggests $\gamma \sim 1200$, which is within a factor of a few of the value estimated above. Thus, we conclude that the high frequency turn down is an expected consequence of IC cooling. This break in the energy spectrum of the relativistic electrons will produce a corresponding break in the spectrum of the IC photons. For relativistic particles, the final energy $\left(E_{\mathrm{f}}\right)$ of an IC photon is related to its initial energy $\left(E_{\mathrm{i}}\right)$ by $E_{\mathrm{f}}=\gamma^{2} E_{\mathrm{i}}$. For an OB-type star, the stellar photon distribution peak occurs $\sim 10 \mathrm{eV}$. This implies that the IC photons resulting from scattering off electrons with $\gamma \sim 10^{3}$ or greater will have energies in excess of $10 \mathrm{MeV}$ - the hard X-ray and gamma ray regimes. Such high energy photon production in CWBs has been examined by other authors e.g. Benaglia \& Romero (2003, and references therein). An additional point is that since $\mathrm{d} \gamma \propto \gamma^{2}$, the higher energy electrons cool most rapidly. This causes the electron distribution to "bunch-up" near $\gamma_{\text {flow }}$, and may help to explain why a mono-energetic fit works well. A model with an evolving value of $\gamma$ will be explored by Pittard et al. (in preparation).

\subsection{Modelling the MERLIN images of WR 147}

The spatial distributions of radio emission at both 5 and $1.6 \mathrm{GHz}$ from the model in Fig. 16 are shown in the top panels of Fig. 17. At $5 \mathrm{GHz}$ the peak intensity of thermal emission from the WR stellar wind and the synchrotron emission from the collision region are similar, and the OB-star companion is clearly visible. At $1.6 \mathrm{GHz}$ the synchrotron emission is much brighter than the WR star stellar wind, and the OB-star wind is barely visible.

To appreciate how these model intensity distributions compare with the MERLIN observations shown in Williams et al. (1997), we used the AIPS subroutine UVCON to generate visibilities appropriate for a MERLIN "observation" of our models. In addition to the array co-ordinates, system noise estimates were included in the calculation by including the performance characteristics of the MERLIN telescopes e.g. antenna efficiencies, system temperatures etc. The resulting visibilities were then imaged and deconvolved using the same procedure as in Williams et al. (1997), giving the "simulated" observations shown in the lower panels of Fig. 17. The remarkable similarity between these images and the observations presented in Williams et al. (1997) is striking. However, here the $5-\mathrm{GHz}$ peak intensity of the collision region is a little lower than that of the WR stellar wind, and the WR star is a little too bright at $1.6 \mathrm{GHz}$. The WR star also appears to be a little more compact than shown in Williams et al. (1997), but we attribute these minor differences to our simple spherical, isothermal model of the stellar winds. Note that the OB star is not visible in the simulated observations. This is because the observations are essentially the intensity distribution (the upper panels) convolved with the interferometer beam and the emission from the OB star is then sufficiently dispersed that it is no longer visible.

One of our hopes in examining the simulated images was to help constrain the inclination angle. The observations shown in Fig. 17 are for an inclination of $0^{\circ}$. At an inclination angle of $30^{\circ}$, the synchrotron emission is spread out over a much larger area and has a much lower surface brightness than in the observations for $i=0^{\circ}$, and shown in Williams et al. (1997). As from the spectral modelling, this leads us to believe that the inclination of the system is quite low, between $0^{\circ}$ and $30^{\circ}$. This is contrary to the conclusions of Pittard et al. (2002) where larger inclination angles were required to recover the extended $\mathrm{X}$-ray emission. However, an alternative explanation for this is that the stellar X-ray emission is extended on larger scales than previously thought (see Skinner et al. 2002).

\section{Summary and future directions}

In this first paper we have modelled the thermal and synchrotron emission from early-type binary systems with a strong wind-wind collision. We have used appropriate simplifying assumptions to make this initial investigation tractable. In particular, we have generated models of wide systems without considering IC cooling, and have assumed that the relativistic particle energy distribution is a power-law up to infinite energies and is spatially invariant throughout the shocked gas. We have also assumed that the magnetic field is highly tangled so that the synchrotron emission is isotropic.

We have demonstrated the importance of considering extended emission and absorption regions, as opposed to treating these in a point-like manner as in previous work. We have also demonstrated the importance of synchrotron self-absorption, the Razin effect, and the free-free opacity of the shocked gas in these systems. With our rather simple model we have been able to model both the spectrum and the spatial distribution of radio emission from WR 147 remarkably well. However, our simple model fails to address the apparent high frequency cutoff in the synchrotron spectrum, due to the current lack of a high energy cutoff in the relativistic electron energy distribution. We note that this cutoff, which suggests a characteristic value of $\gamma \sim 1200$, can be naturally explained by IC cooling.

Clearly if we are to realistically model the observed radio emission from CWBs we will need to calculate the evolution of the relativistic particle energy spectrum as the particles are advected downstream. This scenario will be addressed in a follow-up paper (Pittard et al. , in preparation) where we plan to introduce a finite maximum energy for the relativistic particles and allow this to change along the shock front, as expected for variable IC cooling by photons from the OB star. The inclusion of IC cooling will enable the calculation of the evolution of the energy spectrum of the relativistic particles downstream of the shock acceleration region. Similarly the effect of Coulomb cooling on the lower energy relativistic particles will be incorporated. The emission spectrum of mono-energetic particles can then be convolved with the energy spectrum of the particles to determine the total emission. 

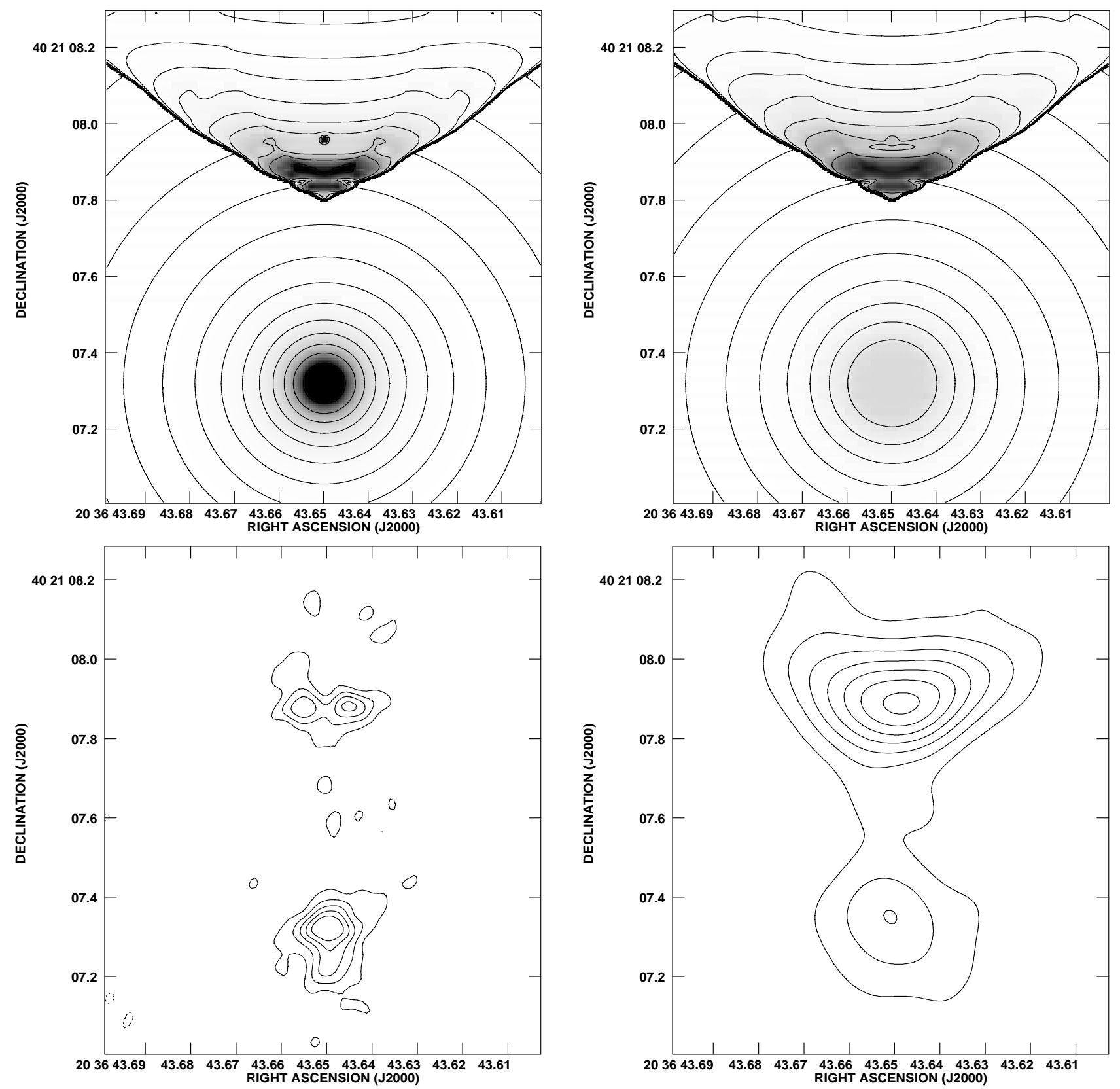

Fig. 17. Intensity distributions at 4.8 (left) and $1.6 \mathrm{GHz}$ (right) of our WR 147 model at $0^{\circ}$ inclination. The top panels are the model intensity distributions at each frequency. The greyscale and contour range are the same in both images. The stellar wind of the OB-star companion is visible, particularly at $5 \mathrm{GHz}$, along with the WR star wind and the wind-wind collision region. The large contour range was used to show that the stellar wind of both stars extends far beyond the greyscale range. The lower images are simulated MERLIN observations generated using the model intensity distributions shown at the top, and the same $u-v$ distribution and beam sizes as Williams et al. (1997). The beams are circular, of diameter 57 and 175 mas at 5 and $1.6 \mathrm{GHz}$ respectively. The contour levels at the two frequencies are also the same as those in Williams et al. (1997). The similarities to the observations of Williams et al. (1997) is striking, most particularly the emission from the wind-collision region.

With these additional mechanisms it will be possible to investigate the high frequency cut-off, such as that apparent in WR 147, and to more accurately model the low frequency cut-off when coulombic cooling is important. Addressing these processes is vital if we are to model smaller binary systems where the electron energy spectrum is highly variable, largely due to the IC cooling from stellar UV photons. This is certainly the case in the proto-typical CWB system WR 140, where it is clear the relative timescales for shock acceleration of electrons, IC cooling, coulombic cooling and adiabatic expansion

are varying dramatically throughout the 7.9-yr orbit. At the very least, these effects, along with radiative shock cooling, need to be considered in any realistic model of WR 140.

Acknowledgements. We would like to thank John Dyson, Tom Harquist, Tony Moffat and Andy Pollock for stimulating discussions related to this work, and the anonymous referee for raising several interesting issues arising in our original manuscript. SMD would like to thank the University of Leeds, UK for their hospitality during a number of visits, and JMP is grateful for hospitality received at DRAO, Canada. JMP is supported by a PDRA position from 
PPARC, and LK has been supported by the NRC Women in Science and Engineering Program. This research has made use of NASA's Astrophysics Data System Abstract Service.

\section{Appendix A: Radiative transfer scheme}

The emergent intensity $I_{i}$ of the radiation field from an increment $\Delta s$ of path length through an emitting and absorbing medium, in the absence of scattering, is given by

$I_{i}=I_{i-1} \mathrm{e}^{-\alpha_{i} \Delta s}+\frac{\varepsilon_{i}}{\alpha_{i}}\left(1-\mathrm{e}^{-\alpha_{i} \Delta s}\right)$,

assuming the absorption $\alpha_{i}$ and emission $\varepsilon_{i}$ coefficients are piecewise constant, and $i=1 \ldots n$. Starting with $I_{1}$, and applying this scheme to each increment of path length in turn, we can derive the emergent intensity $I_{n}$ along the line of sight. In general, the increment of path length $\Delta s$ need not be constant, as long as the emission and absorption coefficients are constant within a given $\Delta s$.

This scheme forms the basis of the radiative transfer code used in this paper. Images are derived by calculating the emergent intensity on a number of lines of sight which pass through a grid of emission and absorption coefficients defined in axissymmetric, cylindrical polar coordinates. The geometry of the problem is shown in Fig. A.1. It is assumed, without any loss of generality, that the line of sight which passes through the origin of the coordinates on the model grid also passes through the origin of the coordinates on the plane of the sky, and that the projection of the line of sight onto planes of constant $z$ is parallel to the line $\phi=0$.

The cell $j, k$ on the model grid is bounded by

$(j-1) \Delta r \leq r<j \Delta r$ and $(k-1) \Delta z \leq z<k \Delta z$

where $\Delta r$ and $\Delta z$ are, respectively, the width and height of a cell in the $r, z$ plane. The grid consists of $N_{r}$ cells in the $r$ direction by $N_{z}$ cells in the $z$ direction. The domain covered by the grid is therefore

$r<R ; 0 \leq z<Z$

where $R=N_{r} \Delta r$ and $Z=N_{z} \Delta z$. The $z$ axis of this grid is inclined at an angle $\theta$ to the plane of the sky, on which we define a co-ordinate system $u, v$. A line of sight through the grid is therefore defined by $u, v$ and $\theta$. All lines of sight are normal to the $u, v$ plane.

It is convenient to define a point along a line of sight in terms of its azimuthal angle $\phi$ in the frame of the grid of emission and absorption coefficients. The co-ordinates on this grid of the point $\phi$ along the line of sight $u, v, \theta$ are given by

$r=\frac{u}{\sin \phi}$

$z=\frac{v}{\cos \theta}+u \frac{\tan \theta}{\tan \phi}$.

The point, $\phi_{0}$, at which the line of sight enters the grid is given by

$\phi_{0}=\min \left\{\sin ^{-1} \frac{u}{R}, \tan ^{-1}\left(-\frac{u}{v} \sin \theta\right)\right\}$.
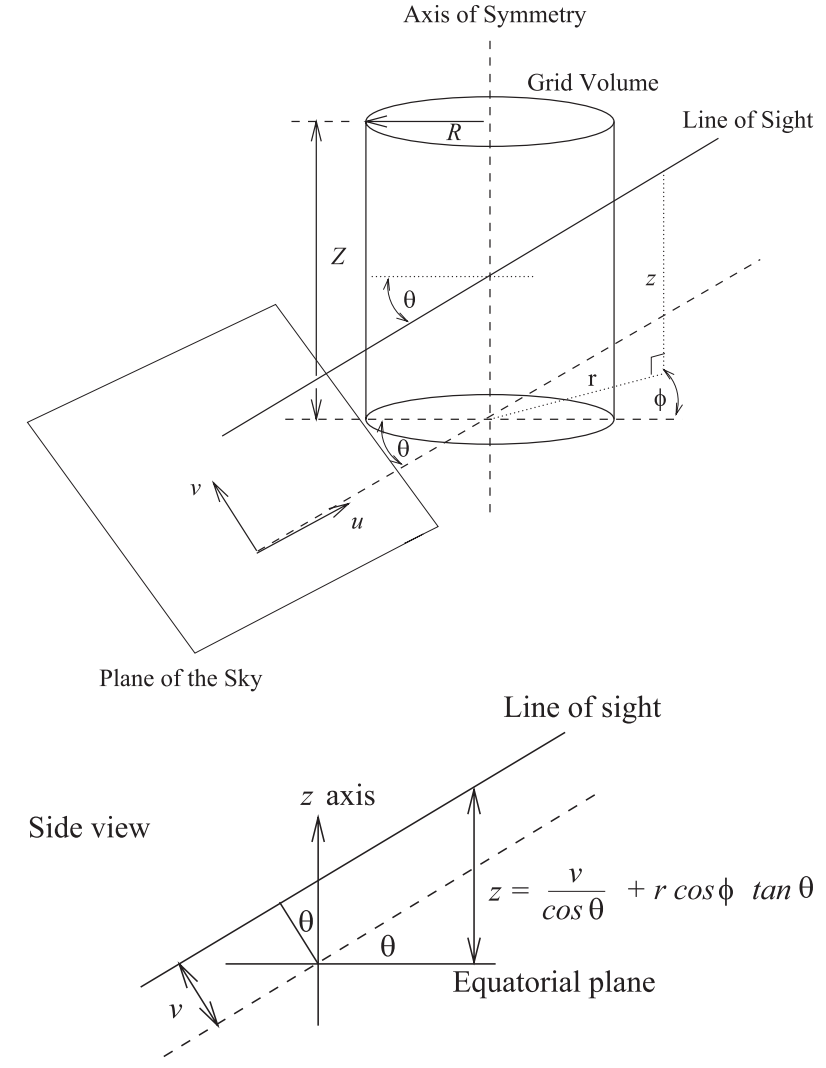

Plan view

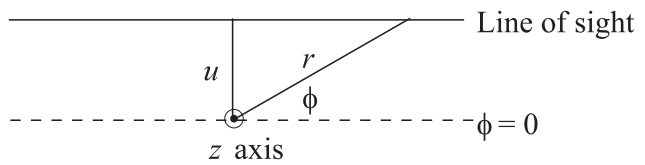

Fig. A.1. Geometry for solving a line of sight through a grid in cylindrical polar coordinates.

The value of $\phi_{0}$ can be used to determine the indices $(j, k)$ of the first grid cell which encounters the line of sight, using Eqs. (A.2), (A.4), and (A.5). The indices of the next cell are determined from the minimum of $\phi_{r}$ and $\phi_{z}$, which are the values of $\phi$ corresponding to the boundaries with the next cells in the $r$ and $z$ directions respectively. The path length through the current cell is then given by

$\Delta s=u \sqrt{1+\tan ^{2} \theta}\left(\frac{1}{\tan \phi_{1}}-\frac{1}{\tan \phi_{0}}\right)$

where $\phi_{1}$ is the point at which the line of sight crosses the next cell boundary.

For a given line of sight, we now have the grid indices of the first cell which encounters the line, the indices of the next cell, and the path length in the current cell. Having obtained the emission and absorption coefficients from the appropriate grid cell, we can update the intensity on the given line of sight using Eq. (A.1). This process is repeated for a line of sight until it leaves the grid (i.e. if $i>N_{r}$ or $j>N_{z}$ ). 


\section{References}

Benaglia, P., \& Romero, G. E. 2003, A\&A, 399, 1121

Blandford, R., \& Eichler, D. 1987, PhR, 154, 1

Blondin, J. M., Kallman, T. R., Fryxell, B. A., \& Taam, R. E. 1990, ApJ, 356, 591

Chapman, J. M., Leitherer, C., Koribalski, B., Bouter, R., \& Storey, M. 1999, ApJ, 518, 890

Chevalier, R. A. 1982, ApJ, 259, 302

Churchwell, E., Bieging, J. H., van der Hucht, K. A., et al. 1992, ApJ, 393, 329

Conti, P. S., \& Ebbets, D. 1977, ApJ, 213, 438

Contreras, M. E., \& Rodríguez, L. F. 1999, ApJ, 515, 762

Contreras, M. E., Rodriguez, L. F., Tapia, M., et al. 1997, ApJ, 488, L153

Donati, J.-F., Babel, J., Harries, T. J., et al. 2002, MNRAS, 333, 55

Donati, J.-F., Wade, G. A., Babel, J., et al. 2001, MNRAS, 326, 1265

Dougherty, S. M., Pittard, J. M., Coker, R., et al. 2003, in Rev. Mex. Astron. Astrofis. Conf. Ser., 56

Dougherty, S. M., \& Williams, P. M. 2000, MNRAS, 319, 1005

Dougherty, S. M., Williams, P. M., \& Pollacco, D. L. 2000, MNRAS, 316,143

Dougherty, S. M., Williams, P. M., van der Hucht, K. A., Bode, M. F., \& Davis, R. J. 1996, MNRAS, 280, 963

Eichler, D., \& Usov, V. 1993, ApJ, 402, 271

Ellison, D. C., Baring, M. G., \& Jones, F. C. 1995, ApJ, 453, 873

Ellison, D. C., Baring, M. G., \& Jones, F. C. 1996, ApJ, 473, 1029

Ellison, D. C., \& Eichler, D. 1985, Phys. Rev. Lett., 55, 2735

Ellison, D. C., \& Reynolds, S. P. 1991, ApJ, 382, 242

Ginzburg, V. L., \& Syrovatskii, S. I. 1965, ARA\&A, 3, 297

Hornby, J. M., \& Williams, P. F. S. 1966, MNRAS, 131, 237

Hummer, D. G. 1988, ApJ, 327, 477

Jardine, M., Allen, H. R., \& Pollock, A. M. T. 1996, A\&A, 314, 594

Lépine, S., Wallace, D., Shara, M. M., Moffat, A. F. J., \& Niemela, V. S. 2001, AJ, 122, 3407

Leitherer, C., \& Robert, C. 1991, ApJ, 377, 629

Lucy, L. B., \& White, R. L. 1980, ApJ, 241, 300

Mioduszewski, A. J., Dwarkadas, V. V., \& Ball, L. 2001, ApJ, 562, 869
Monnier, J. D., Greenhill, L. J., Tuthill, P. G., \& Danchi, W. C. 2002, ApJ, 566, 399

Moran, J. P., Davis, R. J., Spencer, R. E., Bode, M. F., \& Taylor, A. R. 1989, Nature, 340, 449

Morris, P. W., van der Hucht, K. A., Crowther, P. A., et al. 2000, A\&A, 353,624

Niemela, V. S., Shara, M. M., Wallace, D. J., Zurek, D. R., \& Moffat, A. F. J. 1998, AJ, 115, 2047

Pittard, J. M., \& Corcoran, M. F. 2002, A\&A, 383, 636

Pittard, J. M., \& Stevens, I. R. 1997, MNRAS, 292, 298

Pittard, J. M., Stevens, I. R., Williams, P. M., et al. 2002, A\&A, 388, 335

Rybicki, G. B., \& Lightman, A. P. 1979, Radiative processes in astrophysics (New York: Wiley-Interscience, 393 p.)

Setia Gunawan, D. Y. A., de Bruyn, A. G., van der Hucht, K. A., \& Williams, P. M. 2001, A\&A, 368, 484

Skinner, S. L., Itoh, M., Nagase, F., \& Zhekov, S. A. 1999, ApJ, 524, 394

Skinner, S. L., Zhekov, S. A., Güdel, M., \& Schmutz, W. 2002, ApJ, 572, 477

Stevens, I. R. 1995, MNRAS, 277, 163

Stevens, I. R., Blondin, J. M., \& Pollock, A. M. T. 1992, ApJ, 386, 265

Usov, V. V. 1992, ApJ, 389, 635

van der Hucht, K. A., Williams, P. M., Spoclstra, T. A. T., \& de Bruyn, A. C. 1992, in Nonisotropic and Variable Outflows from Stars, ASP Conf. Ser., 22, 253

White, R. L. 1985, ApJ, 289, 698

White, R. L., \& Becker, R. H. 1995, ApJ, 451, 352

Williams, P. M., Dougherty, S. M., Davis, R. J., et al. 1997, MNRAS, 289, 10

Williams, P. M., van der Hucht, K. A., Pollock, A. M. T., et al. 1990a, MNRAS, 243, 662

Williams, P. M., van der Hucht, K. A., Sandell, G., \& The, P. S. 1990b, MNRAS, 244, 101

Wright, A. E., \& Barlow, M. J. 1975, MNRAS, 170, 41

Zhekov, S. A., \& Skinner, S. L. 2000, ApJ, 538, 808 